\title{
TRIP8b-Independent Trafficking and Plasticity of Adult Cortical Presynaptic HCN1 Channels
}

\author{
Zhuo Huang, ${ }^{1}$ Rafael Lujan, ${ }^{2}$ Jose Martinez-Hernandez, ${ }^{2}$ Alan S. Lewis, ${ }^{3}$ Dane M. Chetkovich,, 3 and Mala M. Shah ${ }^{1}$ \\ ${ }^{1}$ Department of Pharmacology, UCL School of Pharmacy, University College London, London, WC1N 1AX, United Kingdom, ${ }^{2}$ Departamento Ciencias \\ Medicas, Centro de Investigación en Discapacidades Neurológicas (IDINE), Universidad de Castilla-La Mancha, 02006 Albacete, Spain, ${ }^{3}$ Davee Department \\ of Neurology and Clinical Neurosciences and ${ }^{4}$ Department of Physiology, Northwestern Feinberg School of Medicine, Chicago, Illinois 60611-3078
}

Hyperpolarization-activated cyclic nucleotide-gated (HCN) channels are subthreshold activated voltage-gated ion channels. In the cortex, these channels are predominantly expressed in dendrites where they significantly modify dendritic intrinsic excitability as well synaptic potential shapes and integration. HCN channel trafficking to dendrites is regulated by the protein, TRIP8b. Additionally, altered TRIP8b expression may be one mechanism underlying seizure-induced dendritic HCN channel plasticity. HCN channels, though, are also located in certain mature cortical synaptic terminals, where they play a vital role in modulating synaptic transmission. In this study, using electrophysiological recordings as well as electron microscopy we show that presynaptic, but not dendritic, cortical HCN channel expression and function is comparable in adult TRIP8b-null mice and wild-type littermates. We further investigated whether presynaptic HCN channels undergo seizure-dependent plasticity. We found that, like dendritic channels, wild-type presynaptic HCN channel function was persistently decreased following induction of kainic acid-induced seizures. Since TRIP8b does not affect presynaptic HCN subunit trafficking, seizure-dependent plasticity of these cortical HCN channels is not conditional upon TRIP8b. Our results, thus, suggest that the molecular mechanisms underlying HCN subunit targeting, expression and plasticity in adult neurons is compartment selective, providing a means by which pre- and postsynaptic processes that are critically dependent upon HCN channel function may be distinctly influenced.

\section{Introduction}

Voltage-gated ion channels are critical regulators of synaptic potential shapes and integration, neuronal firing patterns and synaptic transmission. The expression and biophysical properties of these often vary among the different neuronal subcellular compartments (Nusser, 2009). The hyperpolarization-activated cyclic nucleotide-gated (HCN) channels are highly localized to cortical and hippocampal pyramidal apical dendrites (Robinson and Siegelbaum, 2003; Biel et al., 2009). Here they influence intrinsic excitability and synaptic potential integration (Robinson and Siegelbaum, 2003; Biel et al., 2009; Shah et al., 2010).

\footnotetext{
Received March 28, 2012; revised Aug. 26, 2012; accepted Sept. 1, 2012.
}

Author contributions: R.L. and M.M.S. designed research; Z.H., R.L., J.M.-H., and M.M.S. performed research; A.S.L. and D.M.C. contributed unpublished reagents/analytic tools; Z.H., R.L., J.M.-H., and M.M.S. analyzed data; M.M.S. wrote the paper.

This work was supported by a European Research Council Starter Independent Grant (StG-2010 260725-IRPHRCSTP; M.M.S.), a Medical Research Council New Investigator Grant (G0700369; M.M.S.), a Spanish Ministry of Education and Science Grant (BFU-2009-08404/BFl; R.L.), a CONSOLIDER Grant (CSD2008-00005; R.L.), and National Institutes of Health Grants (NS064757; A.S.L.) (NS055995, NS059934; D.M.C.). The monoclonal antibodies HCN1 (Clone N70/28) were developed by and obtained from the UC Davis/NIH NeuroMab Facility, supported by NIH Grant U24NS050606, and maintained by the Department of Neurobiology, Physiology and Behavior, College of Biological Sciences, University of California, Davis, CA 95616. We thank S. Martin (UCL Sequencing and Genotyping Facility, UK) for genotyping the transgenic mice.

The authors declare no competing financial interests.

Correspondence should be addressed to Dr. Mala M. Shah, Department of Pharmacology, UCL School of Pharmacy, University College London, London, WC1N 1AX, UK. E-mail: mala.shah@ucl.ac.uk.

DOI:10.1523/JNEUROSCI.1544-12.2012

Copyright $\odot 2012$ the authors $\quad 0270-6474 / 12 / 3214835-14 \$ 15.00 / 0$
The expression and trafficking of postsynaptic HCN channels in neurons is regulated by a protein termed TPR-containing Rab8b interacting protein, TRIP8b (Santoro et al., 2004, 2009, 2011; Lewis et al., 2009, 2011; Zolles et al., 2009; Piskorowski et al., 2011). To date nine alternatively spliced isoforms of TRIP8b have been identified (Lewis et al., 2009; Santoro et al., 2009; Zolles et al., 2009). Although all isoforms affect HCN channel gating, the individual isoforms differentially modulate $\mathrm{HCN}$ subunit surface expression (Lewis et al., 2009; Santoro et al., 2009; Zolles et al., 2009). TRIP8b isoform elimination, though, prevents targeting of HCN subunits to hippocampal and cortical dendrites (Lewis et al., 2011; Piskorowski et al., 2011). Further, changes in TRIP8b expression may also underlie some forms of HCN channel plasticity-induced alterations (Shah et al., 2010). Indeed, one possible mechanism underlying seizure-induced dendritic $\mathrm{HCN}$ channel plasticity may be altered HCN-TRIP8b interaction (Shin et al., 2008; Kanyshkova et al., 2012).

Emerging evidence, however, suggests that HCN1 channels are also located within selective cortical and hippocampal synaptic terminals (Southan et al., 2000; Cuttle et al., 2001; Lujan et al., 2005; Aponte et al., 2006; Bender et al., 2007; Boyes et al., 2007; Huang et al., 2011), where they critically influence synaptic release (Southan et al., 2000; Aponte et al., 2006; Huang et al., 2011). Since HCN1 subunits are present only in the synaptic boutons of a subset of neurons, it suggests that these are actively trafficked to these sites. Certain TRIP8b isoforms may be present in axons and regulate $\mathrm{HCN}$ subunit expression here (Piskorowski et al., 2011; Wilkars et al., 2012). We, therefore, asked whether 
TRIP8b isoforms affect HCN1 subunit localization and function in mature cortical synaptic terminals where they are known to be expressed and influence synaptic transmission (Huang et al., 2011). We show that TRIP8b is not involved in HCN1 subunit targeting to these terminals and that synaptic release from these terminals is still modulated by HCN channels. Further, seizureinduced changes in presynaptic HCN1 channels were not reliant on TRIP8b. These results suggest that the cellular mechanisms underlying subcellular trafficking and plasticity of HCN1 channels are likely to differ among the various neuronal compartments. This compartment selectivity may be crucial for the regulation of information processing and therefore, for physiological processes such as cognition as well as pathological conditions such as epilepsy in which HCN channels play an important role (Robinson and Siegelbaum, 2003; Biel et al., 2009; Shah et al., 2010).

\section{Materials and Methods}

TRIP8b-null mice. TRIP8b heterozygote breeding pairs on a C57BL/6J background (Lewis et al., 2011) were imported to UCL School of Pharmacy (London, UK). These TRIP8b ${ }^{+1-}$ animals were crossed to obtain mixtures of TRIP8b-null mice and wild-type littermates which were used in all experiments. The mice were genotyped using protocols similar to that which have been previously described (Lewis et al., 2011).

Kainic acid-induced seizures. Kainic acid (20 mg/kg; Tocris Bioscience Ltd) was administered to wild-type adult (5- to 6-week-old) 129SVEV mice to induce Class V seizures (as defined by the Racine scale; Racine, 1972), which were terminated $1 \mathrm{~h}$ after onset with the use of sodium pentobarbital (SP; $30 \mathrm{mg} / \mathrm{kg}$, s.c.; Sigma-Aldrich). Control groups were mice that had been treated SP only $(30 \mathrm{mg} / \mathrm{kg}$, s.c.). Brain slices were obtained from mice either $24 \mathrm{~h}$ or 1 week following treatment with kainic acid [status epilepticus (SE) mice] or controls (SP mice). All procedures concerning animals were approved by the UK Home Office.

Slice preparation and electrophysiological experiments. All electrophysiological experiments involving transgenic mice were done blindly. Entorhinal-hippocampal slices were obtained from 5- to 8-week-old TRIP8b-null, wild-type littermates, SE mice or SP mice as described previously (Huang et al., 2009). For electrophysiological recordings, slices were placed in a chamber containing external solution of the following composition (in $\mathrm{mm}$ ): $125 \mathrm{NaCl}, 2.5 \mathrm{KCl}, 1.25$ $\mathrm{NaH}_{2} \mathrm{PO}_{4}, 25 \mathrm{NaHCO}_{3}, 2 \mathrm{CaCl}_{2}, 2 \mathrm{MgCl}_{2}, 10$ glucose, 0.05 6-cyano-7nitroquinoxaline-2,3-dione (CNQX), 0.05 DL-AP5, 0.01 bicuculline, 0.001 CGP 55845; bubbled with $95 \% \mathrm{O}_{2} / 5 \% \mathrm{CO}_{2}, \mathrm{pH} 7.2$. For miniature EPSC (mEPSC) experiments, the external solution was supplemented with $0.001 \mathrm{~mm}$ tetrodotoxin and CNQX and DL-AP5 were omitted. For paired-pulse experiments, only CNQX was omitted from the external solution. Whole-cell current-clamp and voltage-clamp recordings were obtained from the soma and dendrites of entorhinal cortical (EC) layer III neurons. The pipette solution contained the following (in $\mathrm{mM}$ ): 120 $\mathrm{KMeSO}_{4}, 20 \mathrm{KCl}, 10 \mathrm{HEPES}, 2 \mathrm{MgCl}_{2}, 0.2$ EGTA, $4 \mathrm{Na}_{2}$-ATP, 0.3 TrisGTP, 14 Tris-phosphocreatine; $\mathrm{pH}$ was adjusted to 7.3 with $\mathrm{KOH}$. For voltage-clamp recordings $0.015 \mathrm{~mm}$ ZD7288 (4-(N-ethyl- $N$ phenylamino)-1,2 dimethyl-6-(methylamino)pyrimidinium chloride) was added to the internal solution. Pipettes containing any of these internal solutions had resistances of 5-12 $\mathrm{M} \Omega$. In some recordings, Neurobiotin $(0.2 \%, \mathrm{w} / \mathrm{v})$ was included in the intracellular pipette solution. Slices were fixed in $4 \%$ paraformaldehyde and stained with streptavidin Alexa Fluor 488 conjugate $24 \mathrm{~h}$ later as described previously (Cossart et al., 2001).

Current-clamp recordings were obtained using an Axoclamp 2B amplifier (Molecular Devices Ltd), filtered at $10 \mathrm{kHz}$, and sampled at 50 $\mathrm{kHz}$. Voltage-clamp recordings were obtained using an Axopatch 200B amplifier (Molecular Devices Ltd), filtered at $1 \mathrm{kHz}$ and sampled at 10 $\mathrm{kHz}$. Series resistance was usually in the order of $10-30 \mathrm{M} \Omega$ and was $\sim 70 \%$ compensated. Recordings were discarded if the series resistance increased by $>20 \%$ during the course of the recordings. Data were ac- quired using pClamp 8.2 or pClamp 10.0 (Molecular Devices Ltd). $\alpha$ EPSPs were generated by current injection of the order:

$$
A=(t / \tau) * \exp (1-(t / \tau))
$$

where $A$ is the amplitude of the current injected and $\tau$ is the rise time constant. For paired-pulse recordings, tungsten electrodes (Biomedical Instruments) placed in EC layer I $\sim 50-100 \mu \mathrm{m}$ from EC layer III distal dendrites were used to elicit single and $20 \mathrm{~Hz}$ pairs of synaptic potentials in interleaved sequences every $30 \mathrm{~s}$.

All reagents were purchased from Sigma-Aldrich, apart from tetrodotoxin, bicuculline, CGP 55845, DL-AP5 and ZD7288, which were obtained from Abcam Ltd. Biocytin was acquired from Vector Laboratories Ltd and streptavidin Alexa Fluor 488 was procured from Life Technologies.

Sholl analysis. Images of neurons that had been filled with Neurobiotin, fixed with $4 \%$ paraformaldehyde and stained using streptavidin Alexa Fluor 488 conjugate were acquired using a confocal microscope (Zeiss LSM 710) and imported into ImageJ (NIH). Concentric circles at $10 \mu \mathrm{m}$ intervals were generated around the somata (Sholl, 1953). The number of dendrites crossing each circle were counted and the mean and SEM reported (Fig. 1A).

Data analysis of electrophysiological recordings. All current-clamp data were analyzed using pClamp 10.0 software (Molecular Devices Ltd). The input resistance $\left(R_{\mathrm{N}}\right)$ was calculated from $400 \mathrm{~ms}$ hyperpolarizing pulses of $100 \mathrm{pA}$ applied from a holding potential of $-70 \mathrm{mV}$. The $\alpha$ EPSP decay time constants were obtained by fitting the double exponential function:

$$
A_{1} e^{(-t / \tau 1)}+A_{2} e^{(-t / \tau 2)},
$$

where $\tau 1$ and $\tau 2$ represent time constants of the initial and falling phase of the $\alpha$ EPSPs. Since $I_{\mathrm{h}}$ is activated during the falling phase of the $\alpha$ EPSP, only $\tau 2$ was used. The summation ratio of EPSPs was calculated as the ratio of the peak of the fifth EPSP to that of the first EPSP.

mEPSC recordings were analyzed using Mini-analysis program (v6.07, Synaptosoft). Events $>1.5 \mathrm{pA}$ in amplitude (i.e., all events above baseline noise level) and with rise times of $<2 \mathrm{~ms}$ were detected and used for analysis. Decay and rise times as well as amplitudes of these events were obtained by fitting the averaged EPSC with a single exponential equation:

$$
I(t)=A \exp (-t / \tau),
$$

where $I$ is the current amplitude at any given time $(t), \mathrm{A}$ is the peak amplitude of the EPSC, and $\tau$ is the decay time constant.

For paired-pulse experiments, data were analyzed using pClamp 10.0 (Molecular Devices Ltd). Interleaved single EPSCs evoked were subtracted from paired EPSCs to obtain the amplitude of the second EPSC. The paired-pulse ratio (PPR) was then calculated as the amplitude of the second EPSC divided by the first. The square of the coefficient of variation $\left(\mathrm{CV}^{2}\right)$ was calculated as

$$
\mathrm{CV}^{2}=\frac{\mathrm{SD}_{\mathrm{PSC}}^{2}-\mathrm{SD}_{\text {Baseline }}^{2}}{\text { Mean }_{\text {Amp }}^{2}},
$$

where $\mathrm{SD}_{\mathrm{PSC}}^{2}$ is the $\mathrm{SD}$ of the EPSC amplitude squared, $\mathrm{SD}_{\text {Baseline }}^{2}$ is the $\mathrm{SD}$ of the background noise squared, and $\mathrm{Mean}_{\mathrm{Amp}}$ is the mean amplitude of the EPSC.

Group data are expressed as mean \pm SEM. Statistical significance was determined using either paired or unpaired Student's $t$ tests as appropriate. Statistical significance of $p<0.05$ is indicated in all figures.

Electron microscopy. Electron microscopic (EM) examination of immunoreactivity for $\mathrm{HCN} 1$ in the mice entorhinal cortex was performed as described previously using the preembedding immunogold method (Luján et al., 1996). Briefly, free-floating sections were incubated in 10\% NGS diluted in Tris-buffered saline (TBS) for $1 \mathrm{~h}$ at room temperature. Sections were then incubated for $48 \mathrm{~h}$ in anti-HCN1 antibodies (UC Davis/NIH NeuroMab Facility) at a final protein concentration of $2-5 \mu \mathrm{g} / \mathrm{ml}$, diluted in TBS containing $1 \%$ NGS. After several washes in TBS, sections were incubated for $3 \mathrm{~h}$ in goat anti-mouse IgG coupled to $1.4 \mathrm{~nm}$ gold (Nanoprobes) diluted 1:100 in TBS containing $1 \%$ NGS. Following washes PBS, the sections were postfixed in $1 \%$ glutaraldehyde diluted in the same buffer for $10 \mathrm{~min}$. They were 
A Wt and TRIP8b neuron morphology

(i)

Wt

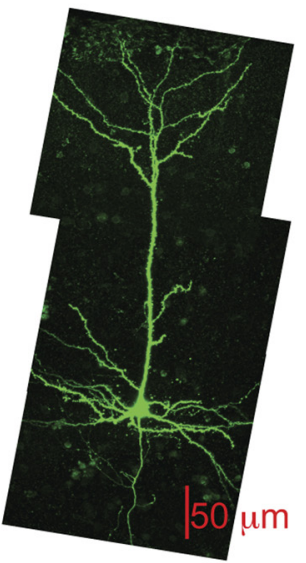

TRIP8b-/-

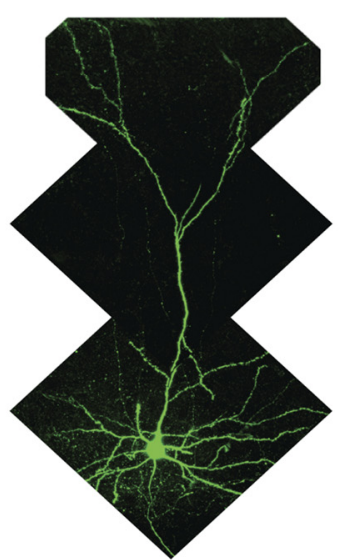

(ii)

Proximal

Dendritic tree

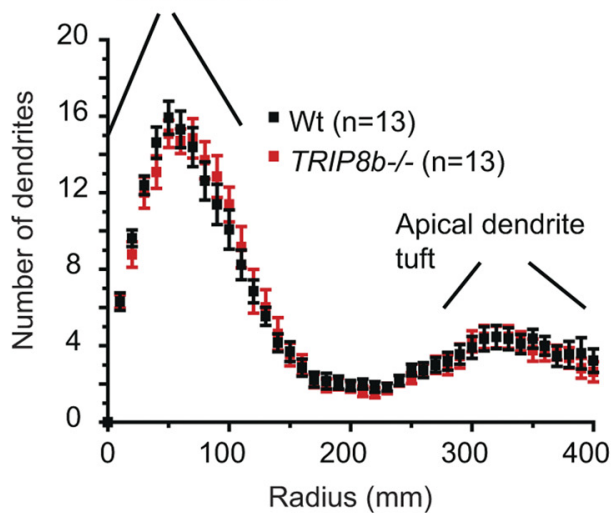

B Wt neuron mEPSC

(i)

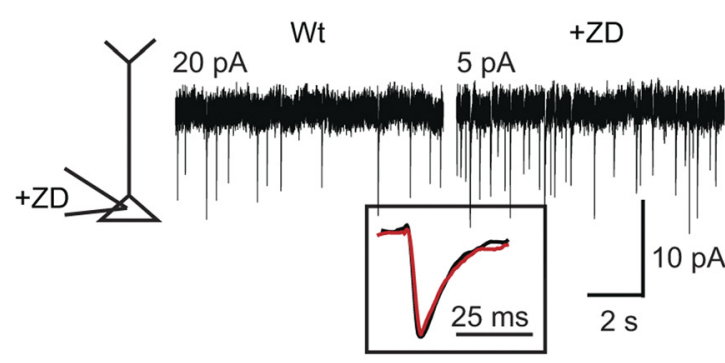

C TRIP8b-/-neuron mEPSC

(i)

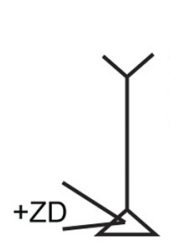

TRIP8b-/-
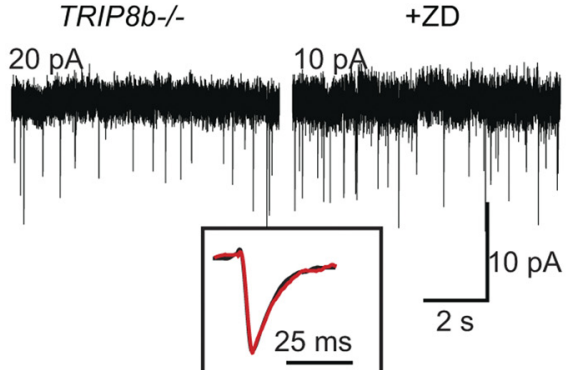

$+Z D$

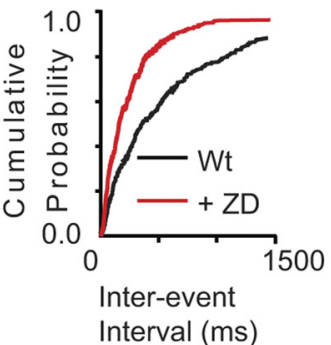

(ii)
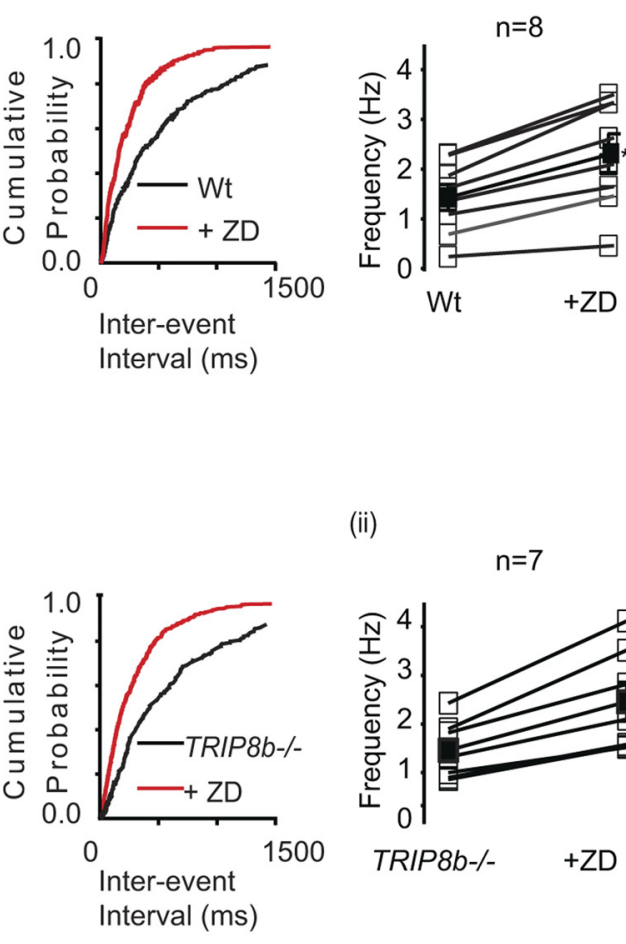

(ii)

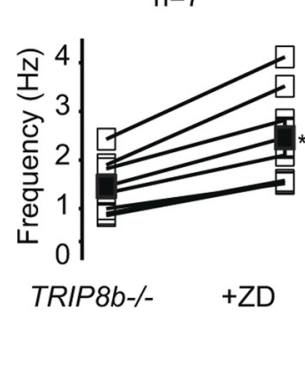

(iii) $\quad-$ Wt $(n=8)$

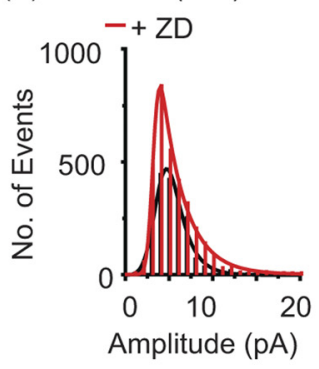

Figure 1. Enhancement of spontaneous excitatory synaptic transmission by presynaptic HCN1 channel inhibition is unaffected by TRIP8b deletion. A(i), Example morphologies of wild-type (Wt)

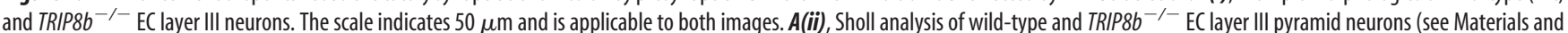
Methods). The proximal dendritic tree represents apical, oblique, and basal dendrites counted within a particular radius. $B(i), C(i)$, Typical mEPSC recordings obtained from wild-type and TRIP8b ${ }^{-\prime-}$ EC layer III neurons before and after $20 \mathrm{~min}$ bath application of the HCN channel blocker, ZD7288 (ZD; $15 \mu \mathrm{m})$. The outward holding values at $-70 \mathrm{mV}$ are shown above the traces. The cumulative probability curves for each of the examples are shown on the right. The average, normalized mEPSCs from the traces under control conditions (black) and after ZD7288 (red) are shown in the insets. The scale shown in $\boldsymbol{B}(\mathbf{i})$ and $\mathbf{C}(\mathbf{i})$ applies to all traces within the respective panels. $\boldsymbol{B}(\mathrm{ii}), \mathbf{C}(\mathrm{ii})$, Graphs depicting the individual (open squares) and the mean (filled squares) $\mathrm{mEPSC}$ frequency obtained from wild-type and $T R I P 8 b^{-/-}$neurons in the absence and presence of ZD7288. The numbers of observations are indicated above the graphs. Significance at ${ }^{*} p<0.05$. B(iii), C(iii), Amplitude histograms of mEPSCs recorded from wild-type and TRIP86 ${ }^{-/-}$neurons under control conditions and following 20 min external application of ZD7288.

washed in double distilled water, followed by silver enhancement of the gold particles with a HQ Silver kit (Nanoprobes). Sections were then treated with osmium tetroxide ( $1 \%$ in $0.1 \mathrm{~m}$ phosphate buffer), block-stained with uranyl acetate, dehydrated in graded series of ethanol and flat-embedded on glass slides in Durcupan (Fluka) resin. Regions of interest were cut at 70-90 nm on an ultramicrotome (Reichert Ultracut E, Leica) and collected on 200- mesh nickel grids. Staining was performed on drops of $1 \%$ aqueous uranyl acetate followed by Reynolds's lead citrate. Ultrastructural analyses were performed using a Jeol-1010 electron microscope.

Quantitative analysis at EM level. To establish the relative abundance of HCN1 gold particles in superficial EC layers, quantification of immunolabeling was performed from $60 \mu \mathrm{m}$ coronal slices as described (Luján 
et al., 1996). For each of three animals, three samples of tissue were obtained (nine total blocks). Electron microscopic serial ultrathin sections were cut close to the surface of each block because immunoreactivity decreased with depth. Randomly selected areas were captured at a final magnification of $45,000 \times$, and measurements covered a total section area of $\sim 2000 \mu \mathrm{m}^{2}$. Dendritic shafts, dendritic spines and axon terminals were assessed for the presence of immunoparticles. The percentage of immunoparticles for $\mathrm{HCN} 1$ at postsynaptic and presynaptic sites was calculated in superficial layers for each animal group.

\section{Results}

Presynaptic HCN channels modulate spontaneous synaptic transmission in TRIP8b-null slices

We have recently shown that HCN1 channels are present in certain terminals synapsing onto mature EC layer III neurons, where they critically influence basal and evoked glutamatergic synaptic transmission (Huang et al., 2011). Indeed, inhibition of presynaptic HCN channels by the pharmacological blocker ZD7288 (15 $\mu \mathrm{M})$ or ablation of HCN1 channels selectively enhanced the frequency of miniature excitatory postsynaptic potentials onto EClayer III pyramids by restricting $\mathrm{Ca}^{2+}$ entry via T-type $\mathrm{Ca}^{2+}\left(\mathrm{Ca}_{\mathrm{V}} 3\right)$ channels (Huang et al., 2011). To investigate whether TRIP8b isoforms may be involved in targeting of HCN subunits to these particular synaptic terminals, we made acute EC-hippocampal slices from adult (5- to 8-week-old) wild types and TRIP8b-null (TRIP8b ${ }^{-/-}$ mice) (Lewis et al., 2011), and recorded from EC layer III neurons. The morphology of wild-type and TRIP $8 b^{-/-}$neurons was found to be not dissimilar (Fig. 1A). mEPSC recordings were obtained at a holding potential of $-70 \mathrm{mV}$ from wild-type and TRIP8 ${ }^{-/-}$neurons in the presence of the tetrodotoxin $(1 \mu \mathrm{M})$ and GABA receptor inhibitors, bicuculline $(10 \mu \mathrm{M})$ and CGP $55845(1 \mu \mathrm{M}$, Fig. $1 B, C)$ as described previously (Huang et al., 2011). In addition, to exclude the effects of postsynaptic HCN channels and to reduce potential spaceclamp errors (Williams and Mitchell, 2008), ZD7288 (15 $\mu \mathrm{M})$ was included in the patch-pipette solution. Incorporating ZD7288 inhibits postsynaptic HCN channels and therefore increases the outward holding current at $-70 \mathrm{mV}$ in wild-type neurons over a period of 10-15 min. This, however, has little effect on mEPSC frequency, amplitude and kinetics (Huang et al., 2011). With ZD7288 in the patch pipette and postsynaptic HCN channels blocked, there was little difference in the outward holding current at $-70 \mathrm{mV}$ between wild-type and TRIP $8 b^{-/-}$neurons. Interestingly, mEPSC frequency, amplitude as well as rise and decay time constants recorded from wild-type and TRIP $8 b^{-/-}$EC layer III neurons were very similar (Fig. $1 B, C$ ), suggesting that presynaptic $\mathrm{HCN}$ channel activity is likely to be comparable in wild-type and TRIP $8 b^{-/-}$entorhinal cortex (EC).

To confirm that presynaptic HCN channel activity is intact, we additionally bath applied ZD7288 (15 $\mu \mathrm{M})$ for 20 min only as this has limited nonspecific effects (Chevaleyre and Castillo, 2002). External application of ZD7288 enhanced mEPSC frequency in wild-type and TRIP8b $b^{-1-}$ neurons by $68.9 \pm 8.1 \%$ $(n=8)$ and $67.9 \pm 4.8 \%(n=7 ; p>0.05$; Fig. $1 B, C)$, respectively. Similar results were obtained if $10 \mu \mathrm{M}$ ZD7288 was applied for either $15 \mathrm{~min}$ or $20 \mathrm{~min}$ (percentage increase in wild-type mEPSC frequency for 15 and $20 \mathrm{~min}=68.7 \pm 1.6 \%(n=6)$ and $69.7 \pm 2.7 \%(n=6)$, respectively). A $10 \mathrm{~min}$ bath application of $10 \mu \mathrm{M}$ ZD7288, however, caused significantly less augmentation in $\mathrm{mEPSC}$ frequency (percentage change in wild-type mEPSC frequency $=58.0 \pm 3.0 \%(n=6 ; p<0.05))$ suggesting that a minimum of $15 \mathrm{~min}$ bath application is required for $10-15 \mu \mathrm{M}$ ZD7288 to have its maximum effect. The mEPSC amplitude, rise and decay time constants were unaffected by external application of ZD7288 (Fig. $1 B, C$ ). These results are in contrast to those obtained using HCN1-null slices (Huang et al., 2011) and suggest that presynaptic $\mathrm{HCN}$ channel function is intact in the absence of TRIP8b. Moreover, since HCN channel inhibitors increased mEPSC frequency in a comparable manner in wild-type and TRIP $8 b^{-/-}$mice, presynaptic HCN channel density is not likely to have been augmented by deletion of all TRIP8b isoforms.

\section{Evoked synaptic transmission modified by presynaptic $\mathrm{HCN}$ channels in TRIP8b ${ }^{-1-}$ slices}

In addition to spontaneous synaptic transmission, presynaptic HCN channels also affect action potential-dependent release in the EC (Huang et al., 2011). If presynaptic HCN channel function is unaffected by TRIP8b, then evoked EPSCs in TRIP8b-null neurons should also be modulated by $\mathrm{HCN}$ channel inhibitors (Huang et al., 2011). To test this, we stimulated EC layer III distal dendrites in the absence of tetrodotoxin to elicit pairs of EPSCs at $20 \mathrm{~Hz}$ (see Materials and Methods; Fig. 2). ZD7288 (15 $\mu \mathrm{M}$ ) was again present in the intracellular solution to inhibit postsynaptic $\mathrm{HCN}$ channels. The extracellular stimulus strength was adjusted such that the first EPSC amplitude was set to values between 50 and $100 \mathrm{pA}$ [average wild-type and TRIP $8 b^{-1-}$ amplitude $=$ $71.2 \pm 4.5 \mathrm{pA}(n=8)$ and $66.0 \pm 4.2(n=9, p>0.05$; Fig. $2 A, B, C$, respectively]. Single EPSCs were routinely induced in between pairs. Subtraction of these from pairs of EPSCs allowed us to determine the amplitude and kinetics of the first and second EPSCs in the pairs and thereby calculate the PPR [Fig. $2 A(i), B(i)]$. There was no difference in the PPR between wildtype and TRIP $8 b^{-1-}$ littermates [wild-type and TRIP $8 b^{-1-}$ $\mathrm{PPR}=0.98 \pm 0.03(n=8)$ and $0.99 \pm 0.04(n=9), p>0.05$; Figure $2 A(i),(i i), B(i),(i i)$, respectively]. The decay and rise time constants of single EPSCs recorded from wild-type and TRIP $8 b^{-1-}$ neurons were similar too (Fig. $2 D, E$ ). Additional bath application of ZD7288 (15 $\mu \mathrm{M})$ for 20 min only increased the amplitude and decreased the rise time constant of the first EPSC in wild-type and TRIP8 $b^{-/-}$neurons to a similar extent [Fig. $2 A(i), B(i), C, E]$. In agreement with a reduction in presynaptic HCN channel activity, added external ZD7288 caused PPR to be reduced in wild-type and TRIP8b ${ }^{-1-}$ neurons to an equal extent [PPR after ZD7288 in wild-type and TRIP8b ${ }^{-/-}=0.74 \pm$ $0.03(n=8)$ and $0.71 \pm 0.04(n=9)$, respectively; Fig. $2 A(i i)$, $B(i i)$. The time course of the reduction in PPR in wild types and TRIP8b ${ }^{-1-}$ following application of ZD7288 was also comparable [Fig. $2 A(i), B(i)$ ]. Further, treatment with ZD7288 caused the $\mathrm{CV}^{2}$ to be lowered to $0.66 \pm 0.07(n=8)$ and $0.68 \pm 0.04(n=9)$ in wild-type and TRIP8b $b^{-/-}$neurons, respectively. As postsynaptic HCN channels were inhibited, the decay time constants were not affected by external application of ZD7288 (Fig. 2D). The equivalent decrease in PPR and $\mathrm{CV}^{2}$ by HCN channel inhibitors in TRIP8 $8 b^{-1-}$ and wild-type neurons provides further evidence that TRIP8b does not affect the function of presynaptic HCN channels.

\section{Postsynaptic HCN channel function in EC layer III neurons is disrupted by TRIP8b}

The above results suggesting that TRIP8b plays no role in the expression or trafficking of presynaptic HCN channel function in the EC were unexpected. Therefore, do the effects of TRIP8b differ within the various cortical substructures or is TRIP8b function neuronal compartment specific? To test this, we compared the postsynaptic somatic and dendritic properties of TRIP8b ${ }^{-/-}$ and wild-type EC layer III neurons using whole-cell current- 
A Wt neurons

(i)

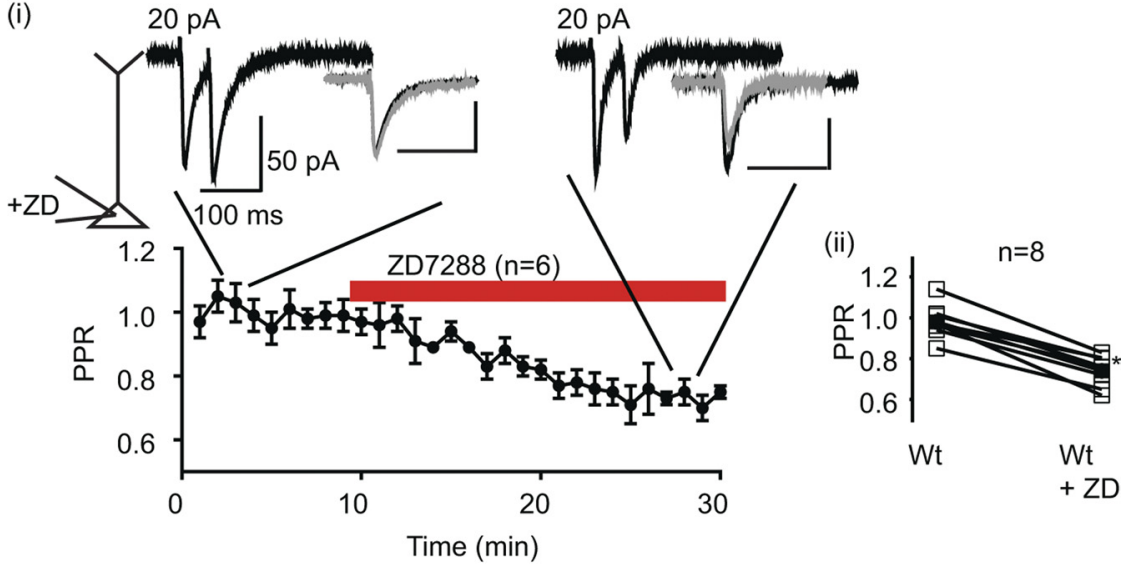

B TRIP8b-/- neurons

ZD7288 $(n=4)$ (i)
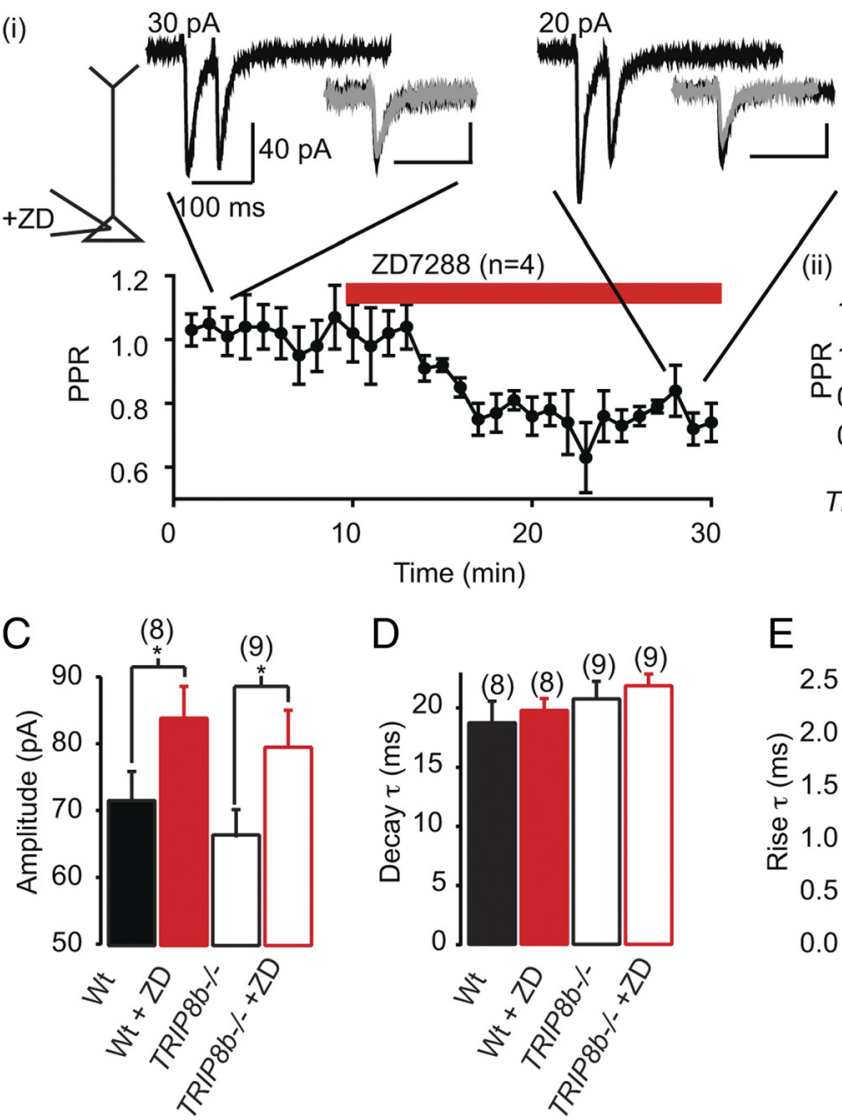

$$
10
$$

Time (min)
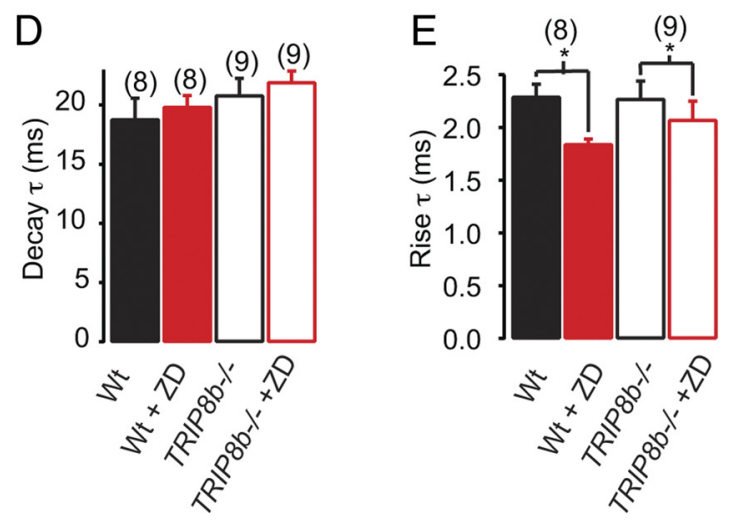

Figure 2. Comparable modulation of PPR by HCN channel inhibitors in wild types and TRIP8b-null neurons. $A(i), B(i)$, Example pairs of EPSCs evoked at a frequency of $20 \mathrm{~Hz}$ in wild-type (Wt) and $T R I P 8 b^{-1-}$ EC layer III pyramids by external stimulation of distal dendrites before (control) and after 20 min external application of ZD7288 (ZD, $15 \mu \mathrm{M})$. The outward holding currents at $-70 \mathrm{mV}$ are stated above the traces. Between each paired pulse, a single stimulus was used to elicit an EPSC. By subtracting this EPSC from the paired EPSCS, the amplitude and shapes of the individual EPSCs were obtained. The insets show the overlaid first EPSC (black) and second EPSC (gray) subtracted traces in the absence and presence of external ZD7288. The time course in the change in PPR caused by 20 min bath application of ZD7288 in 6 wild-type and 4 TRIP86 ${ }^{-/-}$neurons is shown below. The scale bars shown for the control pair of EPSCS in $A(i)$ and $B(i)$ apply to those obtained after application of ZD7288. The vertical and horizontal scale bars for the insets in $A$ (i) are $50 \mathrm{pA}$ and $100 \mathrm{~ms}$, respectively, while those for $B(i)$ represent $40 \mathrm{pA}$ and 100 ms. $\boldsymbol{A}$ (ii), $\boldsymbol{B}$ (ii), Graphs showing the individual (open squares) and mean (filled squares) PPR values obtained from 8 wild-type and 9 TRIP8 ${ }^{-/-}$neurons under control conditions and following 20 min application of $15 \mu \mathrm{m}$ ZD7288. C-E, Bar graphs depicting the amplitudes, decay time constants $(\tau)$, and rise time constants $(\tau)$ of single evoked EPSCs in wild types and TRIP8 ${ }^{-1-}$ neurons in the absence and presence of external ZD7288. The numbers of observations for each group are indicated above the bar. Significance at ${ }^{*} p<0.05$. clamp recordings. In these experiments, ZD7288 was omitted from the patchpipette solution. In addition, the external solution contained glutamate and GABA receptor inhibitors. The somatic and dendritic resting membrane potential (RMP)

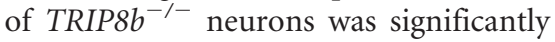
more hyperpolarized than wild types [Fig. $3 A(i)$, (ii); wild-type RMP soma and dendrite RMP $=-69.7 \pm 0.32(n=20)$ and $-68.3 \pm 0.87(n=9)$, respectively; TRIP $8 b^{-1-}$ soma and dendrite RMP = $-79.4 \pm 0.45(n=22)$ and $-78.2 \pm 0.43$ $(n=10)$, respectively]. The dendritic and somatic $R_{\mathrm{N}}$ of TRIP8b ${ }^{-1-}$ neurons was substantially greater than wild types too (Fig. $3 B$ ). The $R_{\mathrm{N}}$ in TRIP8b ${ }^{-/-}$dendrites, particularly, was enhanced fourfold compared with wild types [Fig. 3B(ii)]. In contrast, the difference in wild-type and TRIP $8 b^{-/-}$somatic $R_{\mathrm{N}}$ was less [Fig. $3 B(i i)]$. Thus, despite the more negative RMP, greater numbers of action potentials could be evoked in TRIP8b ${ }^{-/-}$dendrites compared with wild types [Fig. $3 A(i i)]$. At the soma, though, the number of action potentials obtained at the normal RMP in both wild types and TRIP8b $b^{-/-}$ were comparable [Fig. 3A(i)]. These observations are consistent with the reported properties of HCN1-null EC layer III pyramidal cells (Huang et al., 2009) and suggest that TRIP8b deletion significantly reduces postsynaptic HCN channel function. To confirm this, ZD7288 (15 $\mu \mathrm{M})$ was bathapplied for $20 \mathrm{~min}$. Treatment with ZD7288 hyperpolarized the RMP and increased $R_{\mathrm{N}}$ in wild-type soma and dendrites as expected [Fig. $4 A(i), B, C, D(i), E, F]$. In addition, while the numbers of action potentials evoked by depolarizing steps at the soma were similar before and after inhibition of HCN channels if the RMP was not adjusted [Fig. $4 A(i),(i i)$ ], significantly greater spikes could be elicited in dendrites in the presence of ZD7288 than under control conditions, even though the RMP was considerably more negative [Fig. $4 D(i),(i i)$ ]. In contrast, application of ZD7288 had little effect on TRIP8b ${ }^{-/-}$ neuron RMP, $R_{\mathrm{N}}$ or spike number [Fig. $4 A(i i i),($ iv $), B, C, D(i i i),(i v), E, F]$.

The above results also support our previous findings showing that $I_{\mathrm{h}}$ is predominantly dendritic in EC layer III pyramidal neurons (Shah et al., 2004; Huang et al., 2009). Dendritic $I_{\mathrm{h}}$ is vital for postsynaptic integration of EPSPs (Magee, 1999; Williams and Stuart, 2000; Berger et al., 2001; Poolos et al., 2002; Nolan et al., 2004; Shah et al., 2004; Huang et al., 2009). To test whether dendritic EPSP summation is altered in 
A RMP and AP firing

(i) Soma

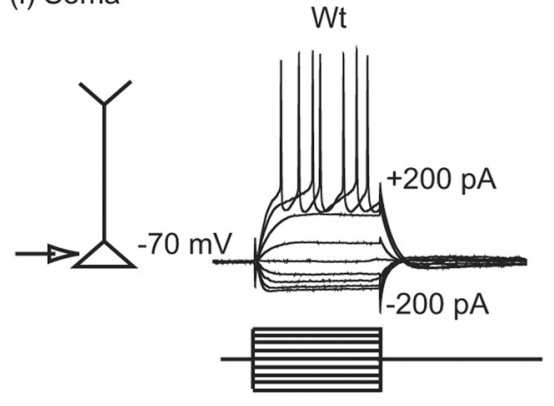

(ii) Dendrite $\quad$ W

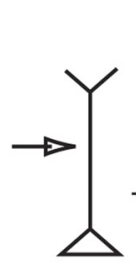

$-69$

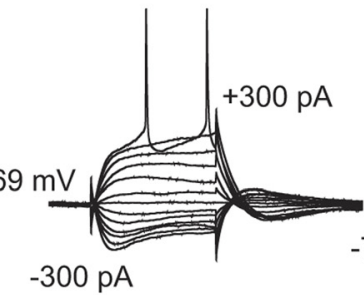

TRIP8b-/-

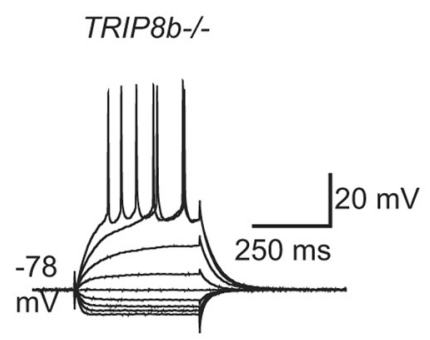

$-78 \mathrm{mV}$

TRIP8b-/-

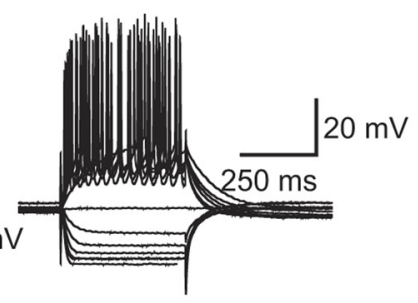

B

$\mathrm{R}_{\mathrm{N}}$

(i)

Soma
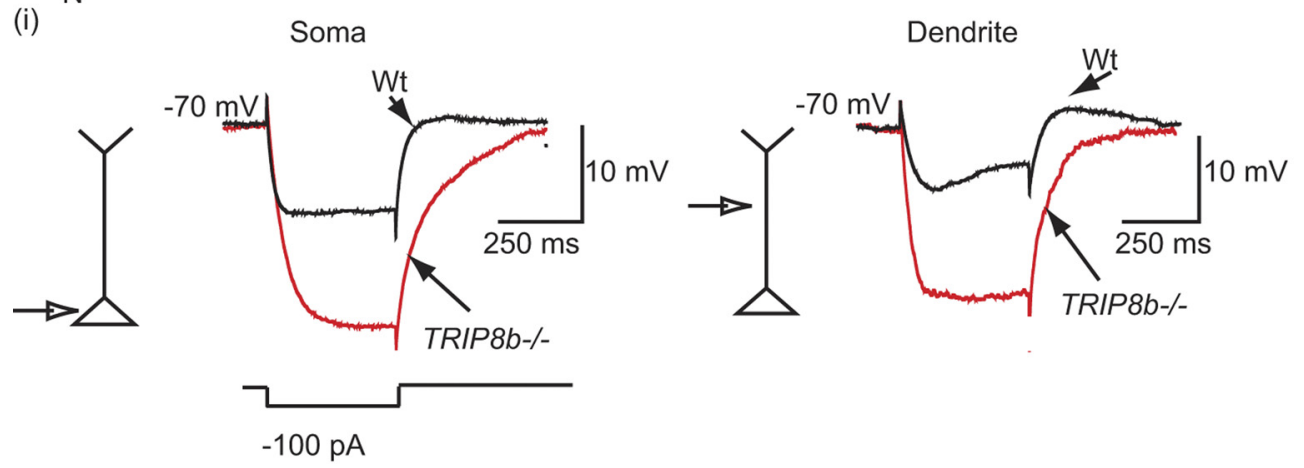

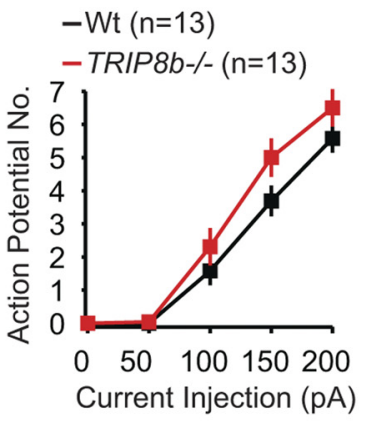

$-W t(n=10)$
$-T R I P 8 b-/-(n=10)$

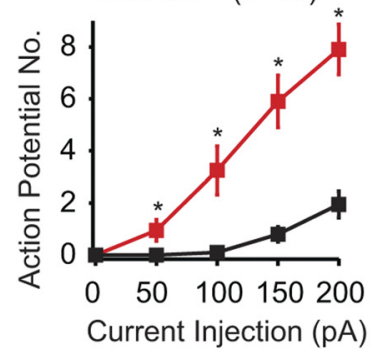

(ii)

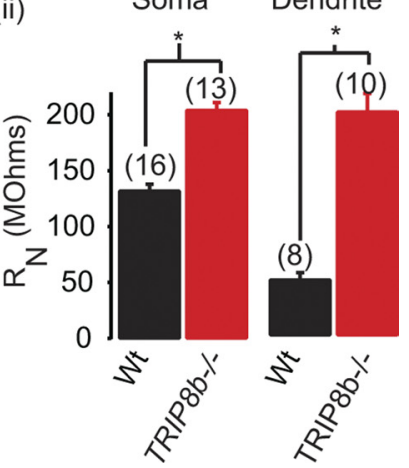

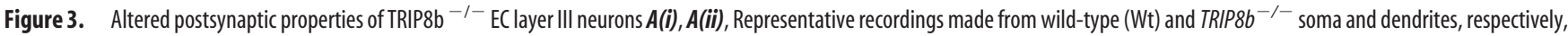
at the normal RMP when a series of $400 \mathrm{~ms}$ hyperpolarizing and depolarizing current steps were applied. The RMP values are indicated on the traces. The average numbers of spikes produced in response to a given depolarizing current injection at the normal RMP are shown on the right. The scale bar shown applies to both traces. $B(i)$, Example wild-type and TRIP8b ${ }^{-1-}$ somatic and dendritic traces obtained when a $400 \mathrm{~ms}, 100 \mathrm{pA}$ hyperpolarizing pulse was applied from a potential of $-70 \mathrm{mV}$. Such traces were used to calculate the $R_{\mathrm{N}}$. $B$ (iii), Bar graph showing the average

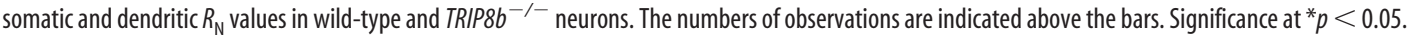

$T_{R I P 8 b^{-1-}}$ neurons, we generated $\alpha$ EPSPs by injecting alpha waveforms locally in dendrites (as previously described by Huang et al., 2009; see Materials and Methods). As expected with reduced dendritic $I_{\mathrm{h}}$ (Huang et al., 2009), single TRIP8b ${ }^{-1-}$ dendritic $\alpha$ EPSP amplitudes were markedly larger than wild types [Fig. $5 A(i)$,(ii)]. In addition, TRIP8b ${ }^{-/-}$dendritic $\alpha$ EPSPs decayed substantially more slowly than wild types [Fig. $5 A(i),(i i)]$. Consequentially, the summation of a train of dendritic $\alpha$ EPSPs either at $20 \mathrm{~Hz}$ or $50 \mathrm{~Hz}$ was significantly greater in TRIP8b ${ }^{-/-}$ neurons than in wild types [Fig. 5B(i),(ii)]. Application of ZD7288 $(15 \mu \mathrm{M})$ for $20 \mathrm{~min}$ increased the amplitude and prolonged the decay of $\alpha$ EPSPs in wild-type dendrites but not in TRIP8b ${ }^{-/-}$dendrites [Fig. 5A(i),(ii)]. Moreover, though the summation of a train of $20 \mathrm{~Hz}$ or $50 \mathrm{~Hz} \alpha$ EPSPs in wild-type dendrites was considerably augmented by ZD7288, $\alpha$ EPSP summation in TRIP8 ${ }^{-1-}$ dendrites was not affected by treatment with this compound [Fig. 5B(i),(ii)]. The electrophysiological characteristics of TRIP8b ${ }^{-1-}$ dendrites and the effects obtained with ZD7288 are very similar to those findings obtained from $\mathrm{HCN1}^{-/-}$EC layer III dendrites (Huang et al., 2009), suggesting that, like in CA1 dendrites (Lewis et al., 2011), deletion of TRIP8b causes severe loss of dendritic HCN channel function in EC layer III pyramids. Thus, the effects of TRIP8b on HCN channels appear to be neuronal compartment selective.

\section{TRIP8b alters dendritic but not presynaptic $\mathrm{HCN} 1$ subunit expression}

We next asked whether the expression pattern of $\mathrm{HCN} 1$ subunits, the predominant form expressed in the EC (Notomi and Shigemoto, 2004; Shah et al., 2004; Huang et al., 2009), is modified by genetic ablation of TRIP8b. To investigate this, we performed immunogold labeling in superficial EC layers (see Materials and Methods for details) using an HCN1 monoclonal antibody that we have previously shown to produce no labeling in HCN1-null tissue (Huang et al., 2011). From each of 3 wild-type and 3 TRIP8b-null mice, 9 tissue blocks were obtained. Ultrathin sec- 
A Soma

(i)<smiles>CC(C)C1CC1</smiles>
$\mathrm{mV}$ Wt

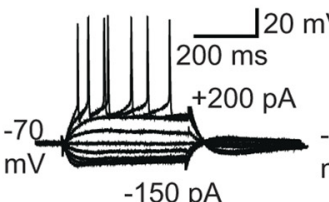
+ZD (HRMP)

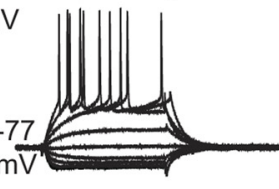

$-150 \mathrm{pA}$ $m \nabla$

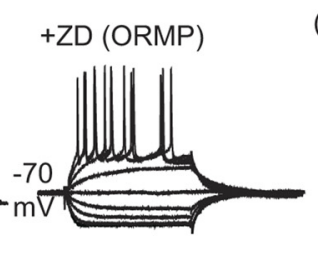

$-W t(n=8)$

-+ ZD (HRMP)

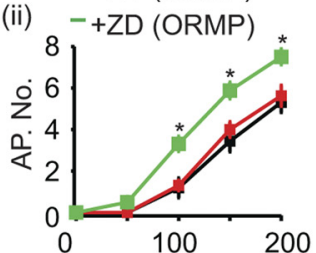

Current injection (pA)

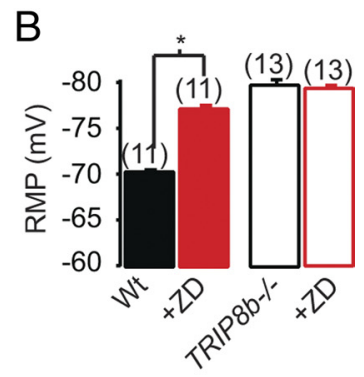

${ }_{\rightarrow}^{\text {(iii) }} Y$

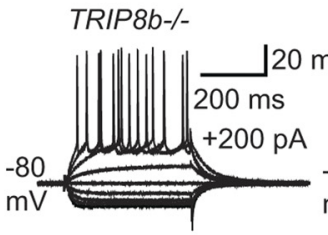

$-150 \mathrm{pA}$

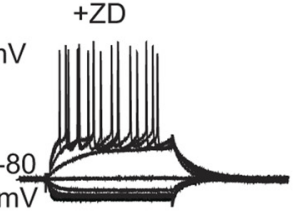

$\mathrm{mV}=$
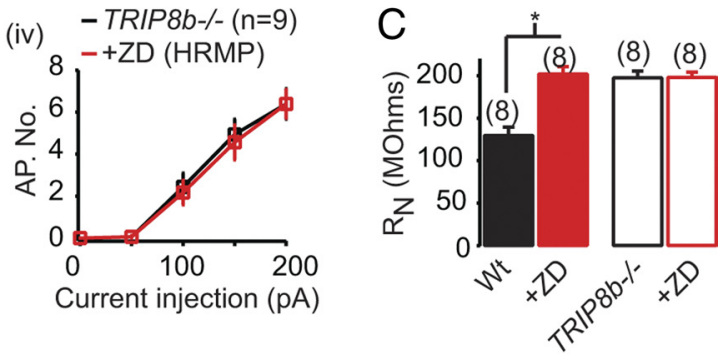

Dendrites

(i)

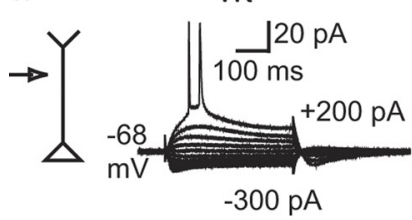

$+\mathrm{ZD}(\mathrm{HRMP})$
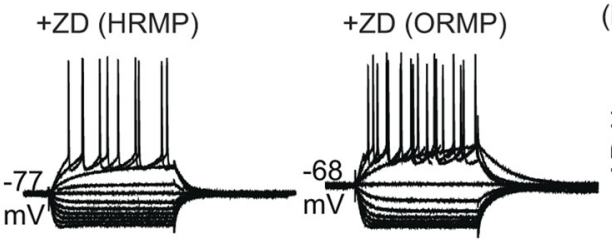

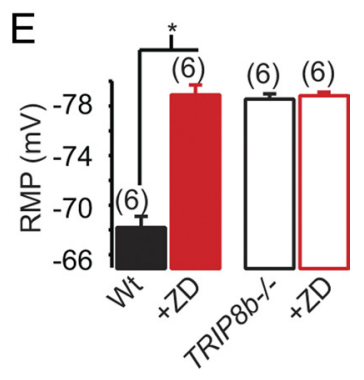

F

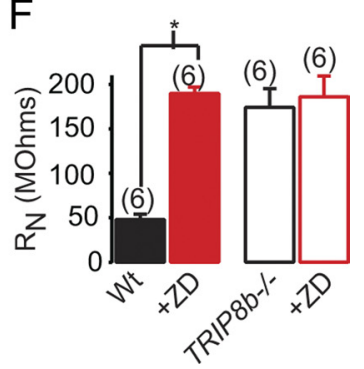

E

(6) (iii)

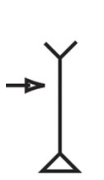

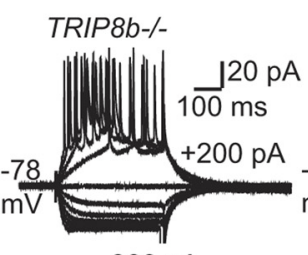

$-300 \mathrm{pA}$

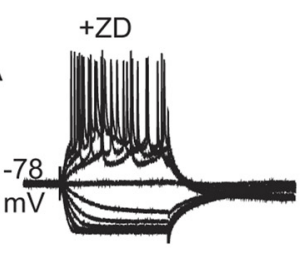

(iv)

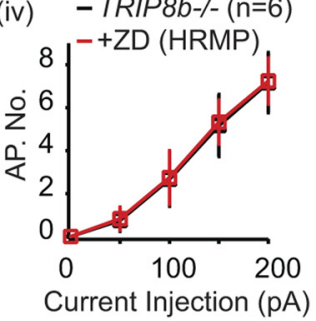

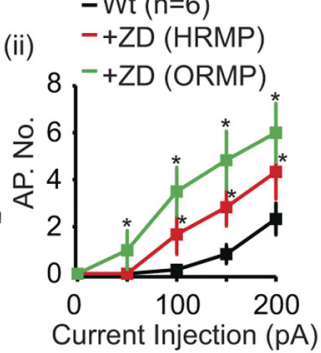

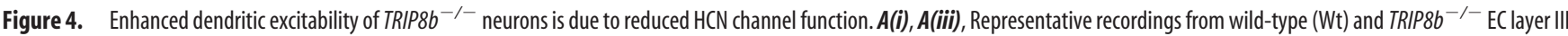
pyramid soma in response to a series of $400 \mathrm{~ms}$ hyperpolarizing and depolarizing steps from $-150 \mathrm{pA} \mathrm{to}+200 \mathrm{pA}$. The RMP values are stated adjacent to the recordings. Traces were obtained under control conditions and following 20 min bath application of ZD7288 (ZD; $15 \mu \mathrm{M})$. ZD7288 caused hyperpolarization of the RMP in wild types and thus recordings were made at the hyperpolarized RMP (HRMP) as well as at the original RMP (ORMP). The scale bars for the control traces in $A$ (i) and $A$ (iii) apply to the other traces shown in those panels. $A$ (ii), $A$ (iv), Graphs depicting the mean and standard error of action potential numbers (AP No.) recorded with a given $400 \mathrm{~ms}$ depolarizing current injection in the absence and presence of ZD7288 in wild-type and TRIP86 ${ }^{-/-}$soma. As ZD7288 hyperpolarized the RMP in wild types, average numbers of spikes produced by a given current injection at the HRMP and ORMP are shown. B, C, Bar graphs to show the RMP and $R_{\mathrm{N}}$ values of wild-type and $T R I P 8 b^{-/-}$soma before and after $20 \mathrm{~min}$ application of ZD7288. The numbers of observations for each group are indicated above the bars. $D(i), D(i i i)$, Example traces obtained from wild-type and TRIP8b ${ }^{-1-}$ EC layer III dendrites at a distance of $150-200 \mu \mathrm{m}$ from the soma in the absence and presence of ZD7288. To obtain the recordings $400 \mathrm{~ms}$ square pulses from $-300 \mathrm{pA}$ to +200 pA were applied. As application of ZD7288 resulted in hyperpolarization of the wild-type RMP, traces were obtained at the HRMP and ORMP. The RMP values are stated beside the traces. The scale bars shown for the controls apply to all traces within the panel. $D\left(\right.$ (ii), $D\left(\right.$ iv), Average spike numbers recorded in wild-type and TRIP86 ${ }^{-/-}$dendrites, respectively, in response to a given $400 \mathrm{~ms}$ depolarizing step from the ORMP under control conditions and following application of ZD7288. E, F, The mean RMP and $R_{\mathrm{N}}$ values obtained in wild-type and $T R I P 8 b^{-/-}$dendrites under control conditions and following the application of ZD7288. The number of observations for each group are indicated above the bar. Significance at ${ }^{*} p<0.05$.

tions were obtained from each of these blocks and particles were detected on dendritic shafts, dendritic spines and axon terminals (Fig. 6A-D). We counted a total of 544 gold particles from wildtype tissue and 466 particles from TRIP $8 b^{-1-}$ tissue. Of these, 443 and 369 were detected on dendritic shafts and terminals of wildtype and TRIP8b ${ }^{-1-}$ tissue, respectively (Fig. 6A,C,E). Although the number of particles counted did not differ (Fig. $6 E$ ), significantly fewer were associated with the plasma membrane in
TRIP8b ${ }^{-/-}$EC dendrites $(47.6 \pm 0.6 \%, n=3$, Fig. $6 C, F)$ than wild types $(80.7 \pm 0.5, n=3, p<0.05$, Fig. $6 A, F)$. Instead, there was a greater intracellular pool in TRIP8b ${ }^{-/-}$EC dendrites $(52.3 \pm 0.6 \%, n=3$, Fig. $6 C, F)$ compared with wild types $(19.3 \pm$ $0.5 \%, n=3, p<0.05$; Fig. $6 A, F)$. In contrast to dendrites, the numbers of particles located on asymmetric axon terminals were comparable in both TRIP8b $b^{-1-}$ and wild types (Fig. 6B,D,E). There was also no difference between plasma membrane associ- 
ated and intracellular particles suggesting TRIP8b has little effect on axonal HCN1 localization (Fig. $6 B, G$ ). No staining was observed if either the primary or secondary antibodies were omitted. These findings re-affirm the notion that TRIP8b regulates dendritic but not presynaptic HCN1 subunit expression in the EC.

\section{Seizure-dependent plasticity of presynaptic $\mathrm{HCN}$ subunits is not reliant on TRIP8b}

A number of studies have now reported that TRIP8b is likely to underlie postsynaptic HCN channel plasticity in a variety of cell types (Shin et al., 2008; Chan et al., 2011; Kanyshkova et al., 2012). In particular, alterations in TRIP8b-HCN channel interaction may underlie seizure-induced changes in dendritic HCN1 subunit expression (Shin et al., 2008; Kanyshkova et al., 2012). Since our results suggest that TRIP8b does not affect trafficking and expression of presynaptic HCN1 subunits, we next asked whether deletion of TRIP8b affects the expression and function of presynaptic HCN channels after generation of seizures.

For these experiments, we used the kainic acid model of SE. Using this model, we have previously shown that SE causes persistent downregulation of dendritic $\mathrm{HCN}$ subunit function in the EC within $24 \mathrm{~h}$ (Shah et al., 2004). This reduction in $\mathrm{HCN}$ channels was associated with hyperexcitability of the EC as interictal spikes can be recorded within $24 \mathrm{~h}$ of kainic acid administration (Shah et al., 2004; Huang et al., 2009). Further, similar to some forms of the human condition (Dawodu and Thom, 2005), minimal cell loss in the EC occurs following termination of kainic acid-generated seizures (Shah et al., 2004). To induce SE (Class V seizures as defined by the Racine scale; Racine, 1972), we administered $20 \mathrm{mg} / \mathrm{kg}$ kainic acid to adult (5- to 6-week-old) mice as previously described (Huang et al., 2009). The seizures were terminated 45-50 min later with sodium pentobarbital. Presynaptic HCN channel function was analyzed at two time points: $24 \mathrm{~h}$ ( $24 \mathrm{~h} \mathrm{SE})$ and 1 week (1 wk SE) following SE induction. Controls were those animals treated with sodium pentobarbital only either $24 \mathrm{~h}$ ( $24 \mathrm{~h}$ $\mathrm{SP}$ ) or 1 week (1 wk SP) prior. These time points were specifically chosen as we have previously shown that postsynaptic $\mathrm{HCN}$ channel function in rat EC layer III neurons is significantly reduced $24 \mathrm{~h}$ and 1 week following SE termination (Shah et al., 2004). Indeed, immunohistochemistry at the light microscopy level also
A Dendritic $\alpha$ EPSPs

(i)

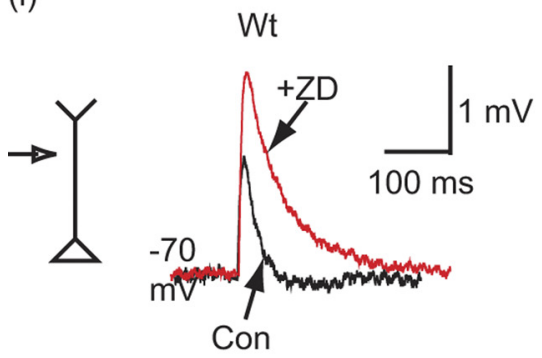

TRIP8b-/-

(ii)
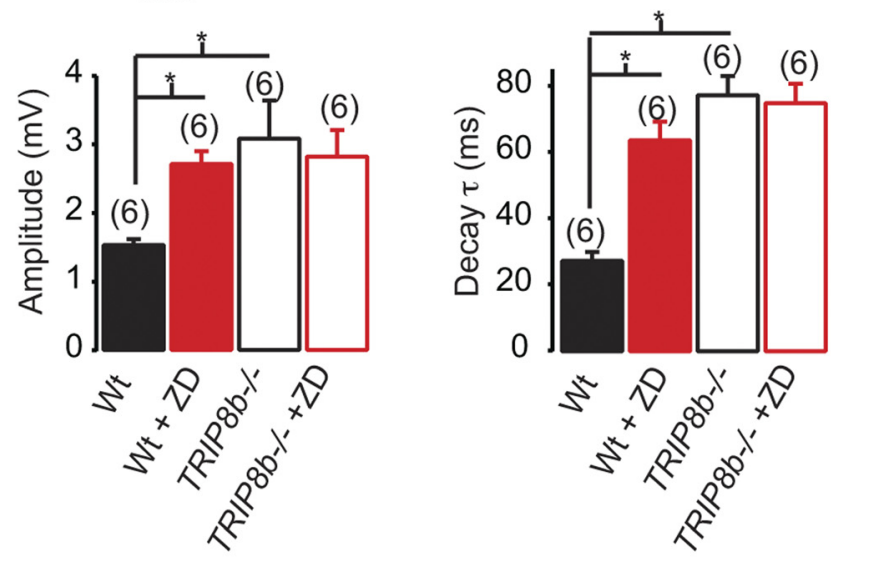

B Dendritic $\alpha$ EPSP Summation

(i)
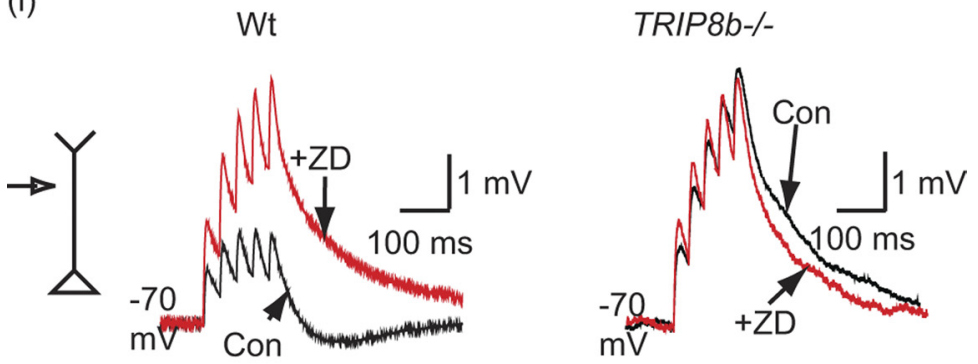

(ii)
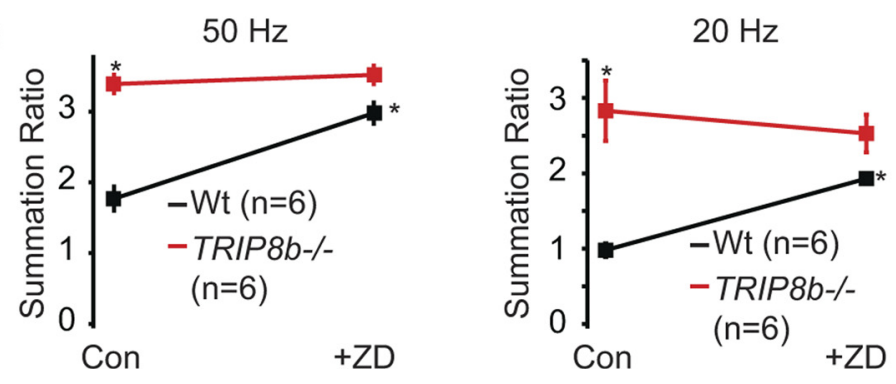

Figure 5. Enhanced postsynaptic EPSP integration due to reduced $\mathrm{HCN}$ channel function in TRIP8b null dendrites. $A(i)$, Example single dendritic $\alpha$ EPSPs obtained at $-70 \mathrm{mV}$ in wild-type (Wt) and TRIP86 ${ }^{-/-}$dendrites at a distance of $100-150 \mu \mathrm{m}$ from the soma under control (con) conditions and following the application of $15 \mu \mathrm{m} Z \mathrm{ZD} 7288$ (ZD). A(ii), Graphs showing the amplitude and decay time constants $(\tau)$ of single $\alpha$ EPSPs before and after ZD7288 in wild types and TRIP8 ${ }^{-/-}$dendrites. The numbers of observations for each group are indicated above the bars. $\boldsymbol{B}(\mathrm{i})$, Representative recordings at $-70 \mathrm{mV}$ of a train of $\alpha \mathrm{EPSPs}$ at a frequency of $50 \mathrm{~Hz}$ in wild-type and TRIP8 ${ }^{-1-}$ dendrites in the absence (con) and presence of ZD7288 (ZD). B(ii), The summation ratios obtained from trains of $\alpha$ EPSPs at frequencies of either $50 \mathrm{~Hz}$ or $20 \mathrm{~Hz}$ under control conditions and after ZD7288 in 6

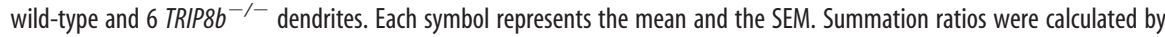
dividing the amplitude of fifth $\alpha$ EPSP by that of the first. The wild-type and TRIP8b ${ }^{-/-}$summation ratios were compared for significance. The ratios obtained in the absence and presence of $Z D 7288$ were also evaluated for significance. $\ln \boldsymbol{A}$ and $\boldsymbol{B}$, significance at ${ }^{*} p<0.05$. 

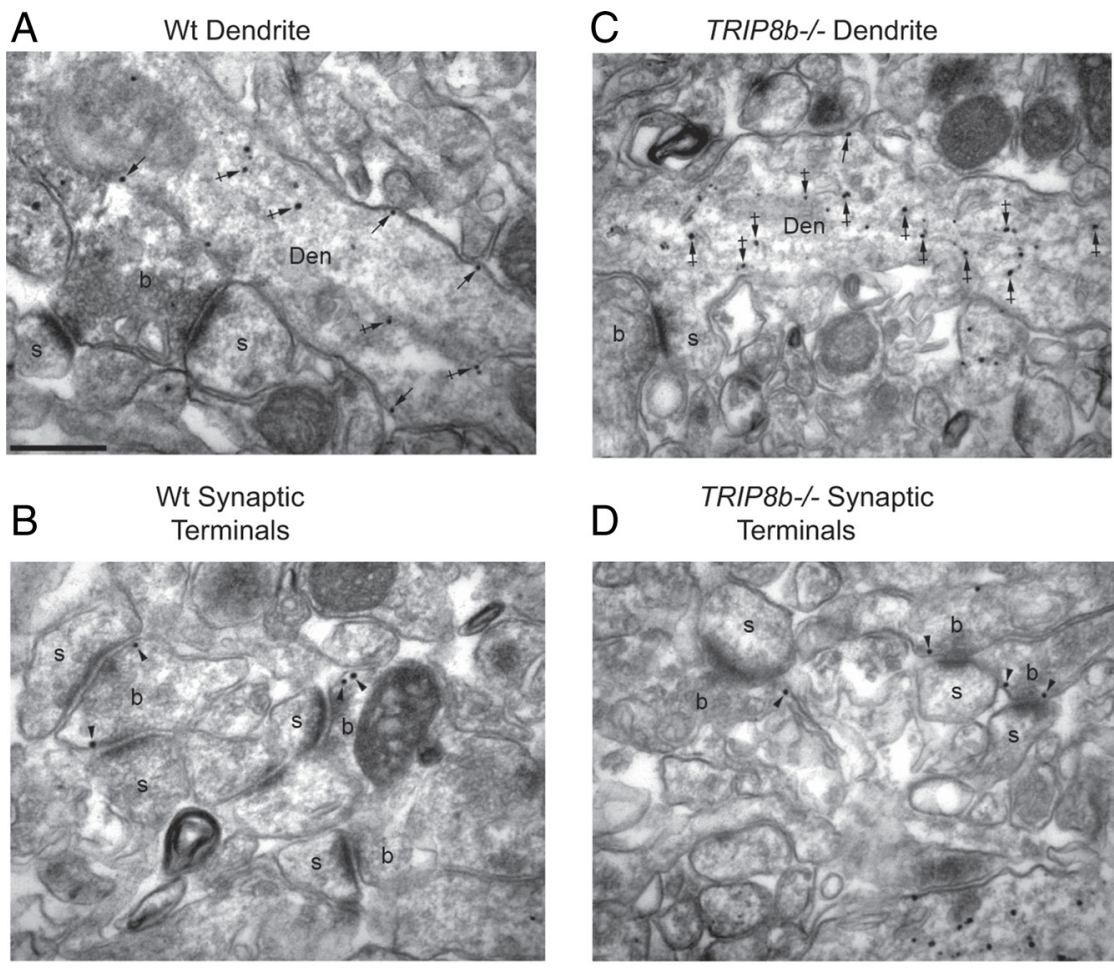

D

TRIP8b-/- Synaptic

Terminals

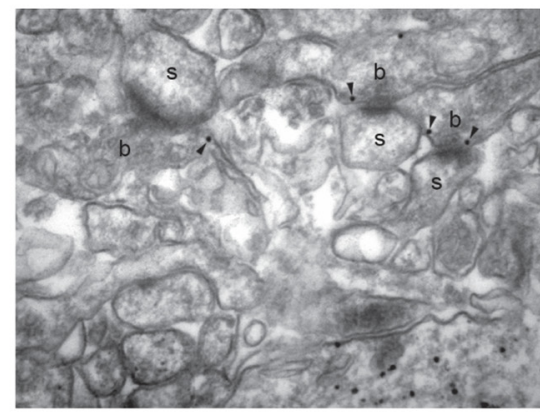

$\mathrm{E}$

Dendrites

\section{Axon}

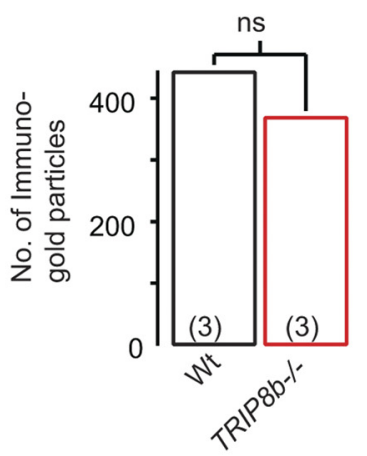

Terminals

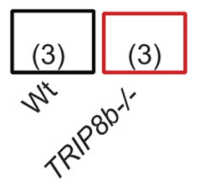

$\mathrm{F}$

$$
\begin{array}{ll}
\text { Plasma } & \text { Intra- } \\
\text { membrane } & \text { cellular }
\end{array}
$$

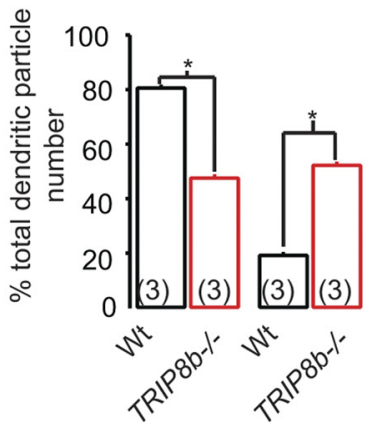

$\mathrm{G}$
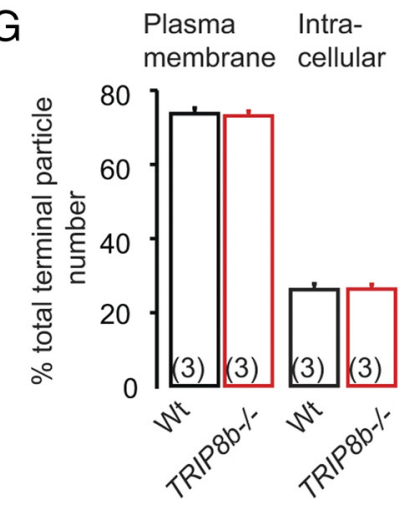

Figure 6. Altered dendritic but not presynaptic HCN1 subunit distribution in TRIP8b EC tissue. A, B, Immunoreactivity for HCN1 in $\mathrm{EC}$ superficial layers in wild-type (Wt) dendrites and synaptic terminals, respectively, as revealed using a preembedding immunogold method. Immunoparticles for HCN1 were located at postsynaptic sites along the extrasynaptic plasma membrane (arrows) of dendritic spines (s) and shafts (Den) establishing synapses with axon terminals (b), as well as associated with intracellular membranes (crossed arrows). A significant proportion of immunoparticles for HCN1 were also detected at presynaptic sites (arrowheads) in axon terminals (b). C, D, HCN1 immunoreactivity in $T R I P 8 b^{-1-}$ EC superficial dendrites and synaptic terminals, respectively. In contrast to wild-type tissue, more particles were located intracellularly (crossed arrows) than along the extrasynaptic plasma membrane (arrows) of dendritic spines (s) and shafts (Den). The scale bar in $\boldsymbol{A}$ represents $0.2 \mu \mathrm{m}$ and applies to all examples shown in $\boldsymbol{A}-\boldsymbol{D}$. $\boldsymbol{E}$, The number of immunogold particles counted from dendrites and synaptic terminals present in

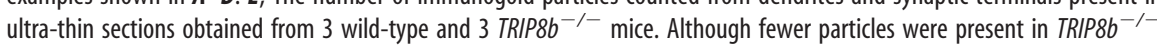

showed a reduction in $\mathrm{HCN} 1$ subunit expression in SE mice sections but not controls (sections from $3 \mathrm{SE}$ and 3 control animals; Fig. 7A). Moreover, electrophysiological recordings showed that the SE dendrite RMP was significantly hyperpolarized compared with that of SP dendrites (Fig. 7B-D). The SE dendrite $R_{\mathrm{N}}$ was also substantially greater than that of SP dendrites (Fig. 7E), resulting in considerably more action potentials being recorded from SE dendrites than SP dendrites, despite the hyperpolarized RMP (Fig. 7B,C). Bath application of ZD7288 $(15 \mu \mathrm{M})$ for $20 \mathrm{~min}$ had little effect on SE dendrites (Fig. 7C-E) but lowered the RMP and enhanced $R_{\mathrm{N}}$ and action potential numbers in SP dendrites (Fig. $7 B, D, E$ ). These results thus affirm our previous findings that SE induces postsynaptic HCN channel plasticity (Shah et al., 2004).

To investigate whether presynaptic HCN channel function, like dendritic $I_{\mathrm{h}}$ (Shah et al., 2004), is reduced, we recorded mEPSCs from SP and SE EC layer III pyramidal somata in the presence of tetrodotoxin and GABA receptor inhibitors as described earlier. In these experiments, ZD7288 (15 $\mu \mathrm{M})$ was incorporated within the patch pipette. Under these conditions, there was no difference in the outward holding current between $24 \mathrm{~h}$ and 1 wk SE and SP neurons. Although mEPSC amplitudes as well as rise and decay time constants were not significantly different (Fig. 8A,B), mEPSC frequency recorded from 24 h or 1 wk SE EC layer III neurons was substantially greater than that obtained from 24 h or $1 \mathrm{wk}$ SP neurons (Fig. $8 A, B)$. These findings were similar to those obtained from HCN1-null neurons (Huang et al., 2011) and suggested that presynaptic HCN channel function may be compromised. Indeed, while external application of ZD7288 (15 $\mu \mathrm{M})$ for $20 \mathrm{~min}$ only markedly enhanced mEPSC frequency in $24 \mathrm{~h}$ and $1 \mathrm{wk}$ SP neurons by $82.0 \pm 19.5 \%[n=6, p<0.05$; Fig. $8 A(i),(i i)]$ and $96.8 \pm 13.3 \%[n=6, p<$ 0.05 ; Fig. $8 B(i),(i i)]$, respectively, it had little effect on mEPSC frequency, amplitude or kinetics recorded from either $24 \mathrm{~h}$ ]Fig. $8 A(i v)-(v i)]$ or 1 wk SE neurons [Fig. $8 B(i v)-(v i)]$.

\section{$\leftarrow$}

dendrites compared with wild types, this was not significant (ns). $\boldsymbol{F}, \mathbf{G}$, Quantification of immunogold particles present on the plasma membrane and in the cytosol of dendrites and axons found in ultra-thin sections produced from 3 wild-type and

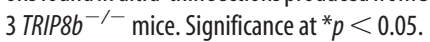


A

$24 \mathrm{hr}$ SP EC

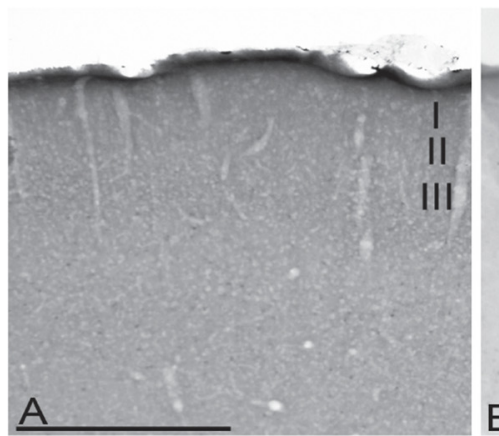

\section{B 24 SP treatment}

(i)<smiles>CC(C)C1CC1</smiles><smiles></smiles>

+ZD (HRMP)

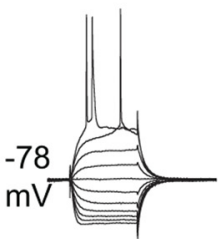

$24 \mathrm{hr}$ SE EC

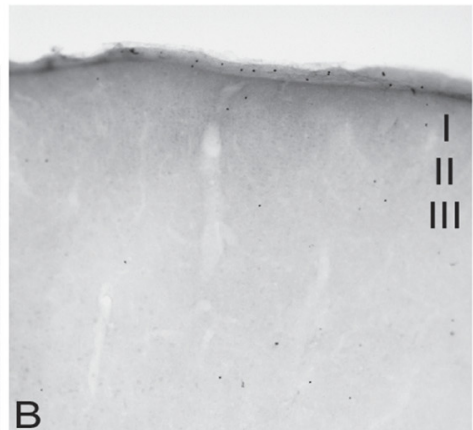

II

III

$-70$

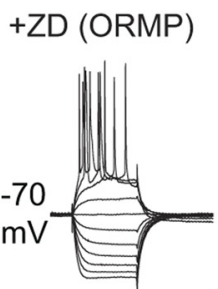

- $24 \mathrm{hr}$ SP $(\mathrm{n}=5)$

- +ZD (HRMP)

(ii) $\quad+$ ZD (ORMP)

(ii) $\quad 1 \mathrm{wk}$ SP $(n=4)$

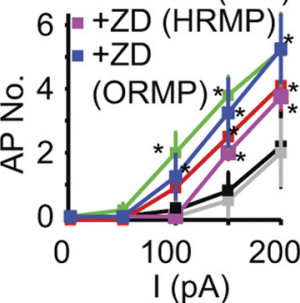

C24 hr SE treatment

(i)<smiles>CC(C)C1CC1</smiles>

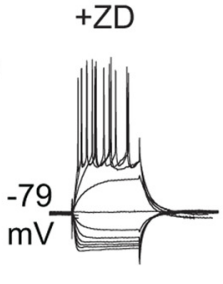

$$
\text { 口24 hr SE (n=3) }
$$$$
\text { a ZD }
$$

(ii) $10 \square$ wk SE $(n=4)$

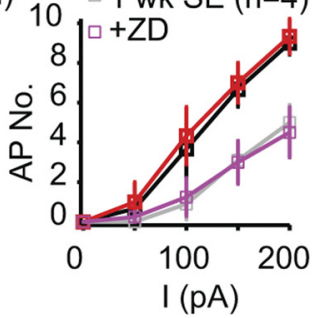

D

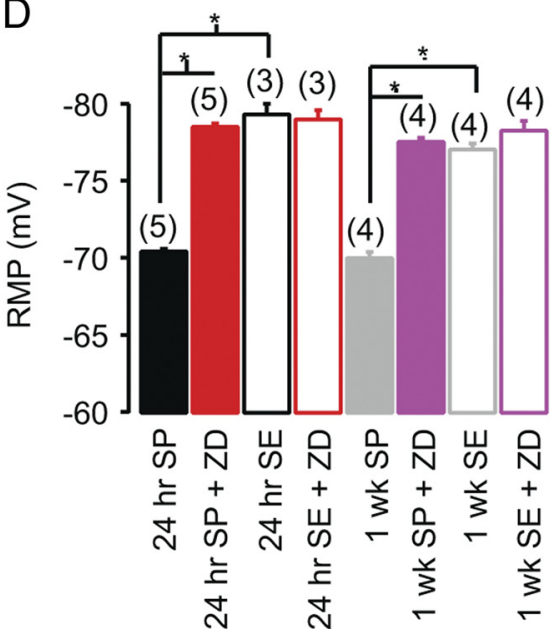

E

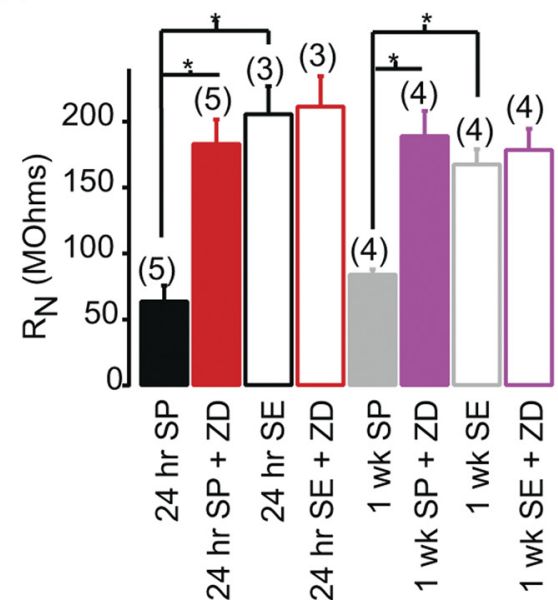

Figure 7. Reduced HCN protein expression following epileptogenesis. $A, \mathrm{HCN} 1$ subunit immunohistochemical analysis using a preembedding immunoperoxidase method in the entorhinal cortex (EC) of animals treated with either SP (30 mg $/ \mathrm{kg}$, s.c.) or kainic acid (20 mg/kg, i.p.) and SP (SE animals) $24 \mathrm{~h}$ prior. Staining patterns are representative of data obtained from 3 different SP and 3 SE mice. Immunoreactivity for HCN1 is strong in EC superficial layers including layers I, II and II from SP mice while it is significantly reduced in the same layers of the SE mice. Scale bar, $500 \mu \mathrm{m}$. $B(i)$, C(i), Example electrophysiological recordings before and after $20 \mathrm{~min}$ application of ZD7288 (15 $\mu \mathrm{m})$ from EC layer III dendrites present in acute slices obtained from $24 \mathrm{~h} \mathrm{SP}$ and $24 \mathrm{~h} \mathrm{SE}$ mice, respectively. The traces were obtained by applying $400 \mathrm{~ms} \mathrm{hyperpolarizing} \mathrm{and} \mathrm{depolarizing} \mathrm{current} \mathrm{steps} \mathrm{from}-300 \mathrm{pA}$ to $+200 \mathrm{pA}$ in increments of $50 \mathrm{pA}$. The RMP of the neurons is indicated next to each recording. Bath application of ZD7288 resulted in hyperpolarization of the RMP in SP neurons and hence the traces obtained at the hyperpolarized RMP (HRMP) as well as the original RMP (ORMP) are shown. The scale bar shown with the first trace applies to all traces within this panel. B(ii), C(ii), Graphs depicting average action potential numbers (AP No.) recorded from 24h SP, 24SE, 1 wkSP, and 1 wkSE neurons in response to depolarizing current injection ( $)$ steps in the absence and presence of ZD7288.D, E, Bar graphs demonstrating the RMP and $R_{N}$ values of SP and SE neurons with and withoutZD7288. The number of observations for each treatment group are shown above each bar. $R_{\mathrm{N}}$ values were obtained by applying a $400 \mathrm{~ms} \mathrm{hyperpolarizing} \mathrm{pulse} \mathrm{from} \mathrm{a} \mathrm{fixed} \mathrm{potential} \mathrm{of}-70 \mathrm{mV}$ in all neurons. Significance at ${ }^{*} p<0.05$. 
A 24 hours treatment

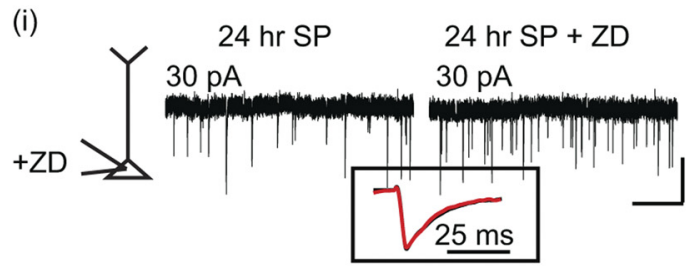

(iv)

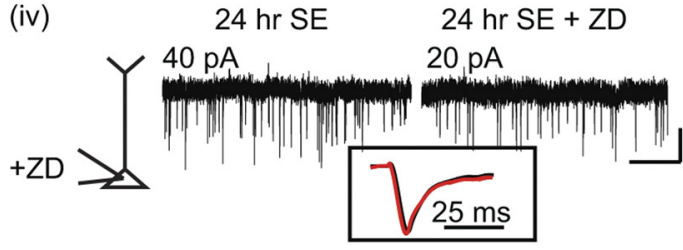

B 1 wk treatment

(i)

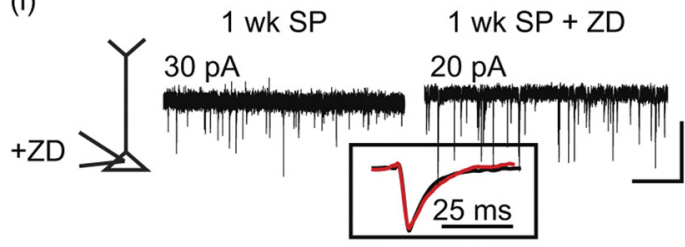

(iv)

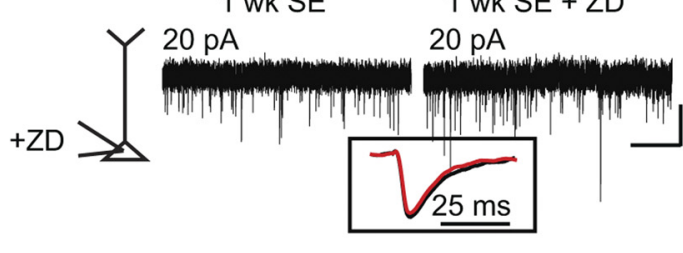

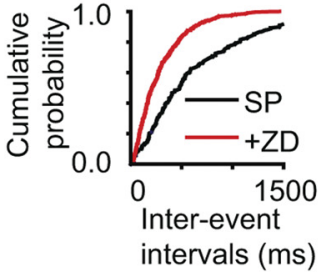

(ii)

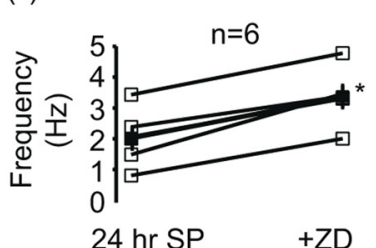

(v)
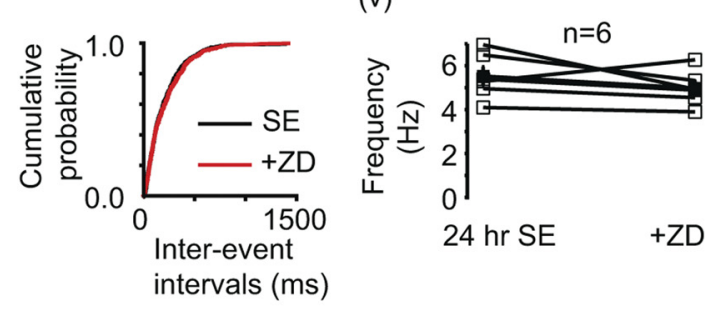

(ii)
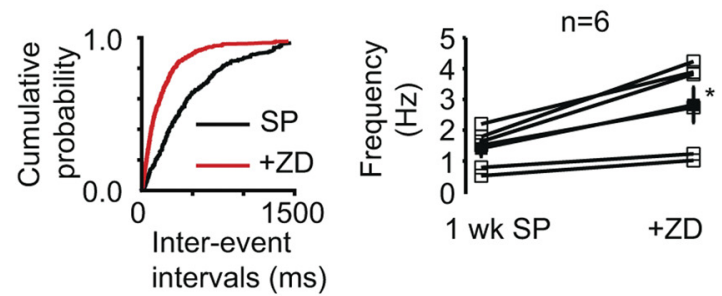

(v)

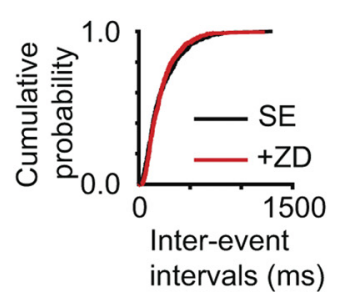

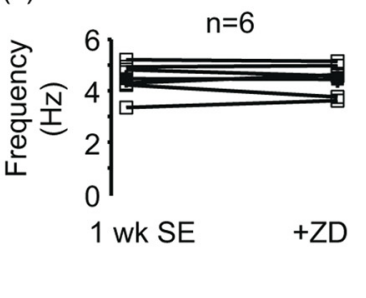
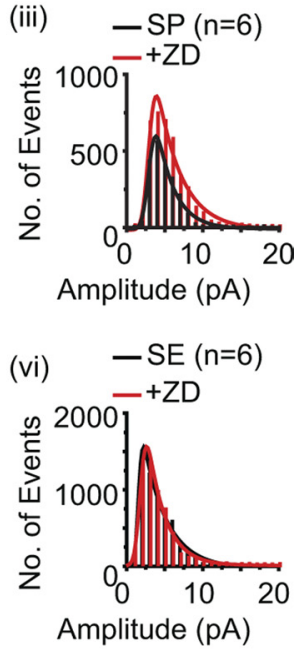

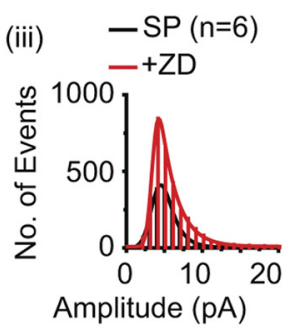

(vi) - SE $(n=6)$

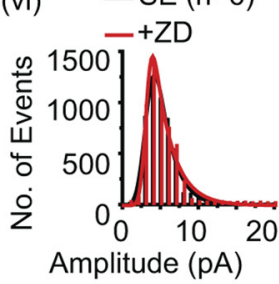

Figure 8. A decrease in presynaptic HCN channel function contributes to enhanced spontaneous excitatory synaptic transmission following epileptogenesis. $A(i)$, (iv), $\boldsymbol{B}(i)$, (iv), Example mEPSC recordings made from EC layer III neurons obtained from mice induced with SE or control (SP) either $24 \mathrm{~h}$ or 1 week prior. The recordings were obtained under control conditions and following 20 min treatment with $15 \mu \mathrm{m} \mathrm{ZD7288} \mathrm{(ZD).} \mathrm{The} \mathrm{values} \mathrm{above} \mathrm{the} \mathrm{traces} \mathrm{represent} \mathrm{the} \mathrm{outward} \mathrm{holding} \mathrm{currents} \mathrm{at}-70 \mathrm{mV}$. The cumulative probability curves for each of the recordings are presented on the right. The average, normalized mEPSC before (black) and after (red) ZD7288 application are shown in the inset. Each horizontal and vertical scale bar for the traces in $A(i), A(i v), B(i)$, and $B(i v)$ denote $100 \mathrm{~ms}$ and $10 \mathrm{pA}$, respectively, and applies to both recordings within each of the panels. $A($ iii), (v), $B(i i)$, (v), Graphs depicting the individual (open squares) and mean (filled squares) $\mathrm{mEPSC}$ frequency in the absence and presence of ZD7288 in $24 \mathrm{~h} \mathrm{SP,} 24 \mathrm{hSE}, 1 \mathrm{wkSP}$, and 1 wk SE neurons, respectively. Numbers of observations for each group are indicated above the graph. $A$ (iiii), (vi), $B$ (iii), $\boldsymbol{B}$ (vi), Amplitude histograms of mEPSCs before and after application of ZD7288 in $24 \mathrm{~h} \mathrm{SP}, 24 \mathrm{~h} \mathrm{SE}, 1$ wk SP and 1 wk SP neurons, respectively. $\boldsymbol{A}$ and $\boldsymbol{B}$, significance at ${ }^{*} p<0.05$.

We also stimulated distal EC layer III dendrites and recorded pairs of evoked EPSCs from the soma. For these particular experiments, tetrodotoxin was omitted from the external solution. The stimulus was adjusted such that the amplitude of the first EPSC was between 50 and $100 \mathrm{pA}$. The PPR obtained from $24 \mathrm{~h} \mathrm{SP}$ $(1.01 \pm 0.05, n=7)$ and $1 \mathrm{wk}$ SP neurons $(1.16 \pm 0.08 ; n=6 ; p>$ $0.05)$ was similar [Fig. $9 A(i), B(i)$ ]. The PPR between $24 \mathrm{~h} \mathrm{SE}$ and SP neurons was also comparable [Fig. 9A(i),(ii)]. Interestingly, the PPR obtained from 1 wk SE neurons $[0.9 \pm 0.09, n=6$; Fig. $9 B(i i)]$ was significantly lower than that acquired from 1 wk SP neurons [1.16 $\pm 0.08 ; n=6, p=0.03$; Fig. 9B(i)]. Since ZD7288 was present in the patch pipette solution, there was no difference between the decay and rise time constants of single EPSCs (data not shown). Additional external application of ZD7288 (15 $\mu \mathrm{M})$ for 20 min only suppressed the EPSC PPR in $24 \mathrm{~h}$ SP [Fig. $9 A(i),($ iii)] and 1 wk SP neurons [Fig. 9B(i)] but had little effect on EPSC PPR obtained from $24 \mathrm{~h}$ SE [Fig. 9A(ii),(iii)] and $1 \mathrm{wk}$ SE neurons [Fig. 9B(ii)]. The $\mathrm{CV}^{2}$ was also substantially reduced in the presence of external ZD7288 to $0.65 \pm 0.03(n=7, p<$ $0.05)$ and $0.67 \pm 0.05(n=6, p<0.05)$ in $24 \mathrm{~h}$ and $1 \mathrm{wk}$ SP neurons, respectively. In contrast, $\mathrm{CV}^{2}$ remained unchanged after bath application of ZD7288 in $24 \mathrm{~h} \mathrm{SE}(0.94 \pm 0.02, n=11)$ and 1 wk SE $(1.01 \pm 0.05, n=6)$ neurons. Moreover, after treatment with external ZD7288, the first EPSC amplitude was markedly increased in $24 \mathrm{~h}$ and $1 \mathrm{wk}$ SP neurons by $18.7 \pm 5.4 \%(n=$ $7, p<0.05)$ and $20.1 \pm 4.4(n=6, p<0.05)$, respectively, but not in $24 \mathrm{~h}(4.3 \pm 5.4, n=11)$ or $1 \mathrm{wk}(1.2 \pm 2.4 \%, n=6) \mathrm{SE}$ neurons. Rise time constants were also considerably decreased in $24 \mathrm{~h}$ and $1 \mathrm{wk}$ SP neurons by $15.1 \pm 2.0 \%(n=7, p<0.05)$ and $12.7 \pm 0.6 \%(n=6, p<0.05)$ but were unchanged in $24 \mathrm{~h}(2.3 \pm$ $3.2 \%, n=11)$ and $1 \mathrm{wk}(3.9 \pm 4.3 \%, n=6)$ SE neurons. Decay time constants, though, were unaffected in either SP or SE neurons fol- 
lowing bath application of ZD7288 (data not shown). The enhanced mEPSC frequency and lowered PPR as well as the lack of effect of external ZD7288 on mEPSCs and PPR in SE neurons suggest that presynaptic HCN channels, like dendritic $\mathrm{HCN}$ channels, are also subject to seizure-induced plasticity. Since presynaptic HCN channel function and expression is unaffected by TRIP8b, this plasticity, unlike that of dendritic HCN1 subunits, is likely to be TRIP8b independent.

\section{Discussion}

In this study, we show that TRIP8b does not influence the expression and function of $\mathrm{HCN}$ channels in synaptic terminals targeting EC layer III pyramidal neurons (Figs. 1, 2,6 ). Like in CA1 and neocortical pyramidal cell dendrites, postsynaptic function of HCN channels was severely reduced in TRIP8b-null EC layer III pyramids (Figs. 3-5), resulting in enhanced dendritic excitability (Figs. 4, 5). This compartmentselective regulation of $\mathrm{HCN}$ channels by TRIP8b was a surprising finding considering that TRIP8b controls the postsynaptic expression of HCN channels in a wide diversity of neuronal subtypes such as hippocampal pyramidal cell dendrites (Santoro et al., 2004, 2009, 2011; Lewis et al., 2009, 2011; Zolles et al., 2009; Piskorowski et al., 2011), globus pallidus neurons (Chan et al., 2011) and thalamic relay neurons (Kanyshkova et al., 2012). Since HCN channels are only found in a subset of glutamatergic and inhibitory synaptic terminals (Beaumont and Zucker, 2000; Southan et al., 2000; Cuttle et al., 2001; Beaumont et al., 2002; Lujan et al., 2005; Aponte et al., 2006; Bender et al., 2007; Boyes et al., 2007; Huang et al., 2011), they are likely to be targeted to these. Our results thus suggest that there may be additional HCN channel binding partners other than TRIP8b that enable targeting of $\mathrm{HCN}$ subunits to presynaptic terminals.

Although our study shows that TRIP8b does not influence HCN1 channel trafficking and expression in mature cortical synaptic terminals (Figs. 1, 2, 6), it is likely to be involved in directing HCN1 subunits to immature perforant path axons that synapse onto hippocampal dentate gyrus granule cells (Wilkars et al., 2012), which also express HCN1 channels (Bender et al., 2007). As these cells mature, HCN1 protein is lost from axons, concordant with an increase in TRIP8b expression (Wilkars et al., 2012). Multiple TRIP8b isoforms exist, some of which reduce HCN expression (Lewis et al., 2009; Santoro et al., 2009; Zolles et al., 2009). Hence, it is possible that mature perforant path axons express TRIP8b isoforms that decrease HCN expression (Wilkars et al., 2012). In agreement with this notion, TRIP8b isoforms that prevent HCN1 expression have been suggested to be located in adult hippocampal CA1 axons (Piskorowski et al., 2011). Further, eliminating all TRIP8b expression results in enhanced HCN1 subunit expression in adult perforant path axons (Wilkars et al., 2012). Therefore, it has been suggested that the principle
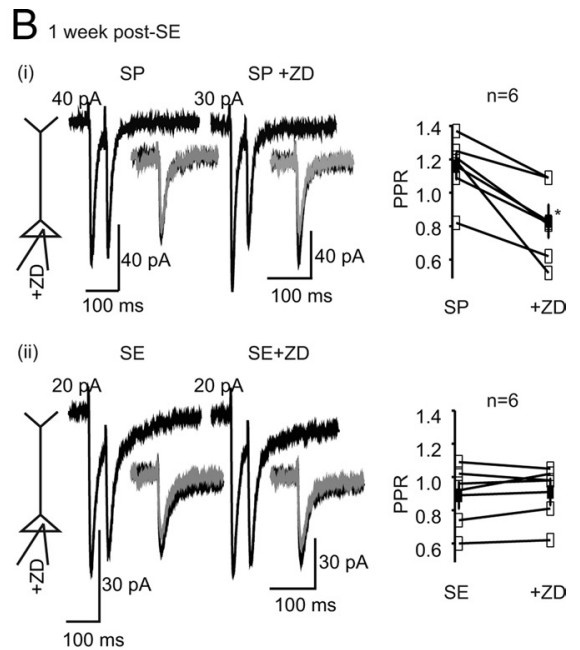

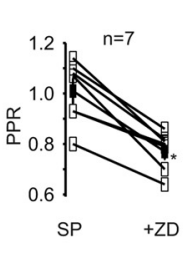

9educed presynaptic HCN channel function following induction of epileptogenesis results in increased evoked syn(open squares) and mean (filled squares) PPRs before and after application of ZD7288 group are indicated on the graph. The scale bars displayed for the pairs and single EPSCs under control conditions apply to the traces illustrating the effects of ZD7288. Significance at ${ }^{*} p<0.05$.

role for TRIP8b is establishing dendritic and eliminating axonal HCN channel expression (Piskorowski et al., 2011; Wilkars et al., 2012). As we found that HCN1 subunit expression was similar in mature TRIP8b-null and wild-type EC synaptic terminals (Fig. 6), one explanation for our findings is that TRIP8b is not expressed in these. Because the origin of the terminals targeting EC layer III neurons that contain HCN channels is as yet unknown (Huang et al., 2011), this question remains to be answered. Regardless, our studies demonstrate that molecular mechanisms independent of TRIP8b are responsible for HCN1 targeting to mature axons.

Changes in HCN-TRIP8 interaction may underlie some forms of HCN channel plasticity, particularly seizure-dependent plasticity of HCN channels (Shin et al., 2008; Chan et al., 2011; Kanyshkova et al., 2012). Because we find that presynaptic HCN channel targeting, expression and function in EC terminals is independent of TRIP8b, the plasticity mechanisms governing presynaptic $\mathrm{HCN}$ channels may differ from those of postsynaptic $\mathrm{HCN}$ channels. Intriguingly, we found postsynaptic and presynaptic $\mathrm{HCN}$ channel function to be persistently reduced in the EC following seizure induction (Figs. 7-9). Additional cellular 
mechanisms such as alterations in transcription/translation and phosphorylation can also cause seizure-dependent changes in HCN channel function (Noam et al., 2011; Shah et al., 2012). Indeed, recent findings suggest that the transcriptional repressor, Neuron Restrictive Silencing Factor (NRSF) binding to the HCN1 promoter is enhanced in the hippocampus and thus HCN1 subunit expression is decreased (McClelland et al., 2011). In support, HCN1 mRNA levels in hippocampal CA1 neurons are lower following seizure induction (Brewster et al., 2002; Powell et al., 2008; Jung et al., 2011). It is therefore possible that, like in the hippocampus, the decline in $\mathrm{HCN} 1$ channel function in the $\mathrm{EC}$ is due to changes at the transcription/translational level. This may explain our observations that both and presynaptic HCN channel function is diminished simultaneously following seizure activity (Figs. 7-9). However, since disrupting the interaction between NRSF and the HCN1 promoter only temporarily restores postsynaptic HCN channel function following SE (McClelland et al., 2011), other post-translational regulatory mechanisms such as phosphorylation must also play a role in the sustained downregulation of HCN channel function after seizures. Certainly, calcineurin and p38 MAPK activity is altered during chronic epilepsy, which is also likely to contribute to the reduction in HCN channel function during epileptogenesis (Jung et al., 2010). Whether similar mechanisms cause alteration in seizure-induced postsynaptic and presynaptic HCN channel function in the EC remains to be elucidated.

In addition to regulating the expression of HCN subunits, TRIP8b also affects the gating of HCN channels. Specifically, the presence of TRIP8b results in a leftward shift of the activation curve such that HCN channels open at more negative potentials (Santoro et al., 2004, 2009; Lewis et al., 2009; Zolles et al., 2009). That presynaptic HCN channel properties are unchanged in TRIP8b-null neurons suggests that the molecular composition and biophysical properties of presynaptic HCN channels differs from those present postsynaptically. Postsynaptic HCN channels, though, are subject to phosphorylation and their properties can be affected by alterations in intracellular calcium concentrations (Biel et al., 2009; Lewis et al., 2010). It remains to be determined whether presynaptic $\mathrm{HCN}$ channels are also modulated by other intracellular signaling molecules. Nonetheless, our results indicate that molecular and cellular mechanisms exist by which pre- and postsynaptic functions that are dependent upon $\mathrm{HCN}$ channel function can be manipulated separately. The ability to individually modulate pre- and postsynaptic HCN channel expression, biophysical characteristics and function may permit stringent regulation of neuronal information processing and thus physiological processes such as cognition and spatial navigation.

\section{References}

Aponte Y, Lien CC, Reisinger E, Jonas P (2006) Hyperpolarizationactivated cation channels in fast-spiking interneurons of rat hippocampus. J Physiol 574:229-243. CrossRef Medline

Beaumont V, Zucker RS (2000) Enhancement of synaptic transmission by cyclic AMP modulation of presynaptic Ih channels. Nat Neurosci 3:133141. CrossRef Medline

Beaumont V, Zhong N, Froemke RC, Ball RW, Zucker RS (2002) Temporal synaptic tagging by $\mathrm{I}_{\mathrm{h}}$ activation and actin: involvement in long-term facilitation and cAMP-induced synaptic enhancement. Neuron 33:601613. CrossRef Medline

Bender RA, Kirschstein T, Kretz O, Brewster AL, Richichi C, Rüschenschmidt C, Shigemoto R, Beck H, Frotscher M, Baram TZ (2007) Localization of HCN1 channels to presynaptic compartments: novel plasticity that may contribute to hippocampal maturation. J Neurosci 27:4697-4706. CrossRef Medline

Berger T, Larkum ME, Lüscher HR (2001) High $\mathrm{I}_{\mathrm{h}}$ channel density in the distal apical dendrite of layer $\mathrm{V}$ pyramidal cells increases bidirectional attenuation of EPSPs. J Neurophysiol 85:855-868. Medline

Biel M, Wahl-Schott C, Michalakis S, Zong X (2009) Hyperpolarizationactivated cation channels: from genes to function. Physiol Rev 89:847885. CrossRef Medline

Boyes J, Bolam JP, Shigemoto R, Stanford IM (2007) Functional presynaptic HCN channels in the rat globus pallidus. Eur J Neurosci 25:2081-2092. CrossRef Medline

Brewster A, Bender RA, Chen Y, Dube C, Eghbal-Ahmadi M, Baram TZ (2002) Developmental febrile seizures modulate hippocampal gene expression of hyperpolarization-activated channels in an isoform- and cellspecific manner. J Neurosci 22:4591-4599. Medline

Chan CS, Glajch KE, Gertler TS, Guzman JN, Mercer JN, Lewis AS, Goldberg $\mathrm{AB}$, Tkatch T, Shigemoto R, Fleming SM, Chetkovich DM, Osten P, Kita H, Surmeier DJ (2011) HCN channelopathy in external globus pallidus neurons in models of Parkinson's disease. Nat Neurosci 14:85-92. CrossRef Medline

Chevaleyre V, Castillo PE (2002) Assessing the role of Ih channels in synaptic transmission and mossy fiber LTP. Proc Natl Acad Sci U S A 99:95389543. CrossRef Medline

Cossart R, Dinocourt C, Hirsch JC, Merchan-Perez A, De Felipe J, Ben-Ari Y, Esclapez M, Bernard C (2001) Dendritic but not somatic GABAergic inhibition is decreased in experimental epilepsy. Nat Neurosci 4:52-62. CrossRef Medline

Cuttle MF, Rusznák Z, Wong AY, Owens S, Forsythe ID (2001) Modulation of a presynaptic hyperpolarization-activated cationic current $\left(\mathrm{I}_{\mathrm{h}}\right)$ at an excitatory synaptic terminal in the rat auditory brainstem. J Physiol 534: 733-744. CrossRef Medline

Dawodu S, Thom M (2005) Quantitative neuropathology of the entorhinal cortex region in patients with hippocampal sclerosis and temporal lobe epilepsy. Epilepsia 46:23-30. CrossRef Medline

Huang Z, Walker MC, Shah MM (2009) Loss of dendritic HCN1 subunits enhances cortical excitability and epileptogenesis. J Neurosci 29:1097910988. CrossRef Medline

Huang Z, Lujan R, Kadurin I, Uebele VN, Renger JJ, Dolphin AC, Shah MM (2011) Presynaptic HCN1 channels regulate Cav3.2 activity and neurotransmission at select cortical synapses. Nat Neurosci 14:478-486. CrossRef Medline

Jung S, Bullis JB, Lau IH, Jones TD, Warner LN, Poolos NP (2010) Downregulation of dendritic HCN channel gating in epilepsy is mediated by altered phosphorylation signaling. J Neurosci 30:6678-6688. CrossRef Medline

Jung S, Warner LN, Pitsch J, Becker AJ, Poolos NP (2011) Rapid loss of dendritic HCN channel expression in hippocampal pyramidal neurons following status epilepticus. J Neurosci 31:14291-14295. CrossRef Medline

Kanyshkova T, Meuth P, Bista P, Liu Z, Ehling P, Caputi L, Doengi M, Chetkovich DM, Pape HC, Budde T (2012) Differential regulation of HCN channel isoform expression in thalamic neurons of epileptic and nonepileptic rat strains. Neurobiol Dis 45:450-461. CrossRef Medline

Lewis AS, Schwartz E, Chan CS, Noam Y, Shin M, Wadman WJ, Surmeier DJ, Baram TZ, Macdonald RL, Chetkovich DM (2009) Alternatively spliced isoforms of TRIP8b differentially control h channel trafficking and function. J Neurosci 29:6250-6265. CrossRef Medline

Lewis AS, Estep CM, Chetkovich DM (2010) The fast and slow ups and downs of HCN channel regulation. Channels 4:215-231. Medline

Lewis AS, Vaidya SP, Blaiss CA, Liu Z, Stoub TR, Brager DH, Chen X, Bender RA, Estep CM, Popov AB, Kang CE, Van Veldhoven PP, Bayliss DA, Nicholson DA, Powell CM, Johnston D, Chetkovich DM (2011) Deletion of the hyperpolarization-activated cyclic nucleotide-gated channel auxiliary subunit TRIP8b impairs hippocampal Ih localization and function and promotes antidepressant behavior in mice. J Neurosci 31:74247440. CrossRef Medline

Luján R, Albasanz JL, Shigemoto R, Juiz JM (2005) Preferential localization of the hyperpolarization-activated cyclic nucleotide-gated cation channel subunit HCN1 in basket cell terminals of the rat cerebellum. Eur J Neurosci 21:2073-2082. CrossRef Medline

Magee JC (1999) Dendritic lh normalizes temporal summation in hippocampal CA1 neurons. Nat Neurosci 2:508-514. CrossRef Medline

McClelland S, Flynn C, Dubé C, Richichi C, Zha Q, Ghestem A, Esclapez M, Bernard C, Baram TZ (2011) Neuron-restrictive silencer factormediated hyperpolarization-activated cyclic nucleotide gated channelo- 
pathy in experimental temporal lobe epilepsy. Ann Neurol 70:454-464. CrossRef Medline

Noam Y, Bernard C, Baram TZ (2011) Towards an integrated view of HCN channel role in epilepsy. Curr Opin Neurobiol 21:873-879. CrossRef Medline

Nolan MF, Malleret G, Dudman JT, Buhl DL, Santoro B, Gibbs E, Vronskaya S, Buzsáki G, Siegelbaum SA, Kandel ER, Morozov A (2004) A behavioral role for dendritic integration: HCN1 channels constrain spatial memory and plasticity at inputs to distal dendrites of CAl pyramidal neurons. Cell 119:719-732. CrossRef Medline

Notomi T, Shigemoto R (2004) Immunohistochemical localization of Ih channel subunits, HCN1-4, in the rat brain. J Comp Neurol 471:241-276. CrossRef Medline

Nusser Z (2009) Variability in the subcellular distribution of ion channels increases neuronal diversity. Trends Neurosci 32:267-274. CrossRef Medline

Piskorowski R, Santoro B, Siegelbaum SA (2011) TRIP8b splice forms act in concert to regulate the localization and expression of HCN1 channels in CA1 pyramidal neurons. Neuron 70:495-509. CrossRef Medline

Poolos NP, Migliore M, Johnston D (2002) Pharmacological upregulation of h-channels reduces the excitability of pyramidal neuron dendrites. Nat Neurosci 5:767-774. Medline

Powell KL, Ng C, O’Brien TJ, Xu SH, Williams DA, Foote SJ, Reid CA (2008) Decreases in HCN mRNA expression in the hippocampus after kindling and status epilepticus in adult rats. Epilepsia 49:1686-1695. CrossRef Medline

Racine RJ (1972) Modification of seizure activity by electrical stimulation. I. After-discharge threshold. Electroencephalogr Clin Neurophysiol 32: 269-279. CrossRef Medline

Robinson RB, Siegelbaum SA (2003) Hyperpolarization-activated cation currents: from molecules to physiological function. Annu Rev Physiol 65:453-480. CrossRef Medline

Santoro B, Wainger BJ, Siegelbaum SA (2004) Regulation of HCN channel surface expression by a novel C-terminal protein-protein interaction. J Neurosci 24:10750-10762. CrossRef Medline

Santoro B, Piskorowski RA, Pian P, Hu L, Liu H, Siegelbaum SA (2009) TRIP8b splice variants form a family of auxiliary subunits that regulate gating and trafficking of HCN channels in the brain. Neuron 62:802-813. CrossRef Medline

Santoro B, Hu L, Liu H, Saponaro A, Pian P, Piskorowski RA, Moroni A, Siegelbaum SA (2011) TRIP8b regulates HCN1 channel trafficking and gating through two distinct C-terminal interaction sites. J Neurosci 31: 4074-4086. CrossRef Medline

Shah MM, Anderson AE, Leung V, Lin X, Johnston D (2004) Seizureinduced plasticity of $\mathrm{h}$ channels in entorhinal cortical layer III pyramidal neurons. Neuron 44:495-508. CrossRef Medline

Shah MM, Hammond RS, Hoffman DA (2010) Dendritic ion channel trafficking and plasticity. Trends Neurosci 33:307-316. CrossRef Medline

Shah MM, Huang Z, Martinello K (2012) $\mathrm{HCN}$ and $\mathrm{K}_{\mathrm{V}} 7(\mathrm{M}-$ ) channels as targets for epilepsy treatment. Neuropharmacology. Advance online publication. Retrieved March 15, 2012. doi:10.1016/j.neuropharm.2012.03.005. CrossRef

Shin M, Brager D, Jaramillo TC, Johnston D, Chetkovich DM (2008) Mislocalization of $\mathrm{H}$ channel subunits underlies $\mathrm{H}$ channelopathy in temporal lobe epilepsy. Neurobiol Dis 32:26-36. CrossRef Medline

Sholl DA (1953) Dendritic organization in the neurons of the visual and motor cortices of the cat. J Anat 87:387-406. Medline

Southan AP, Morris NP, Stephens GJ, Robertson B (2000) Hyperpolarizationactivated currents in presynaptic terminals of mouse cerebellar basket cells. J Physiol 526:91-97. CrossRef Medline

Wilkars W, Liu Z, Lewis AS, Stoub TR, Ramos EM, Brandt N, Nicholson DA, Chetkovich DM, Bender RA (2012) Regulation of axonal HCN1 trafficking in perforant path involves expression of specific TRIP8b isoforms. PLoS One 7:e32181. CrossRef Medline

Williams SR, Mitchell SJ (2008) Direct measurement of somatic voltage clamp errors in central neurons. Nat Neurosci 11:790-798. CrossRef Medline

Williams SR, Stuart GJ (2000) Site independence of EPSP time course is mediated by dendritic $I_{h}$ in neocortical pyramidal neurons. J Neurophysiol 83:3177-3182. Medline

Zolles G, Wenzel D, Bildl W, Schulte U, Hofmann A, Müller CS, Thumfart JO, Vlachos A, Deller T, Pfeifer A, Fleischmann BK, Roeper J, Fakler B, Klöcker N (2009) Association with the auxiliary subunit PEX5R/Trip8b controls responsiveness of HCN channels to cAMP and adrenergic stimulation. Neuron 62:814-825. CrossRef Medline 